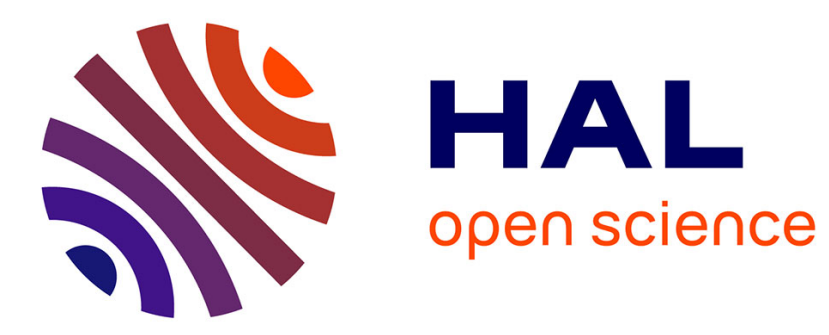

\title{
Axiom directed Focusing
}

Clément Houtmann

\section{To cite this version:}

Clément Houtmann. Axiom directed Focusing. Types for Proofs and Programs, International Conference, TYPES 2008, Mar 2008, Torino, Italy. pp.169-185, 10.1007/978-3-642-02444-3_11 . inria$00212059 \mathrm{v} 3$

\section{HAL Id: inria-00212059 https://hal.inria.fr/inria-00212059v3}

Submitted on 2 Jul 2008

HAL is a multi-disciplinary open access archive for the deposit and dissemination of scientific research documents, whether they are published or not. The documents may come from teaching and research institutions in France or abroad, or from public or private research centers.
L'archive ouverte pluridisciplinaire HAL, est destinée au dépôt et à la diffusion de documents scientifiques de niveau recherche, publiés ou non, émanant des établissements d'enseignement et de recherche français ou étrangers, des laboratoires publics ou privés. 


\title{
Axiom directed Focusing
}

\section{long version}

\author{
Clément Houtmann \\ Université Henri Poincaré Nancy $1 \&$ LORIA*, \\ Campus Scientifique, BP 239 \\ 54506 Vandoeuvre-lès-Nancy Cedex, France \\ Clement.Houtmann@loria.fr
}

\begin{abstract}
Superdeduction and deduction modulo are methods specially designed to ease the use of first-order theories in predicate logic. Superdeduction modulo, which combines both, enables the user to make a distinct use of computational and reasoning axioms. Although soundness is ensured, using superdeduction and deduction modulo to extend deduction with awkward theories can jeopardize essential properties of the extended system such as cut-elimination or completeness w.r.t. predicate logic. Therefore one has to design criteria for theories which can safely be used through superdeduction and deduction modulo. In this paper we revisit the superdeduction paradigm by comparing it with the focusing approach. In particular we prove a focalization theorem for cut-free superdeduction modulo: we show that permutations of inference rules can transform any cut-free proof in deduction modulo into a cut-free proof in superdeduction modulo and conversely, provided that some hypotheses on the synchrony of reasoning axioms are verified. It implies that cutelimination for deduction modulo and for superdeduction modulo are equivalent. Since several criteria have already been proposed for theories that do not break cut-elimination of the corresponding deduction modulo system, these criteria also imply cut-elimination of the superdeduction modulo system, provided our synchrony hypotheses hold. Finally we design a tableaux method for superdeduction modulo which is sound and complete provided cut-elimination holds.
\end{abstract}

Key words: proof theory, superdeduction, focusing, deduction modulo

\section{Introduction}

Computer science and mathematics are both concerned with the construction of formal proofs. These proofs are used as certificates for assertions, theorems or programs since once they have been constructed, they can be easily communicated, replayed and understood. Therefore formal proofs are the highest criterion for trusting a piece of software. The construction of proofs usually relies on the definition of some proof system which contains both a discipline which the user

\footnotetext{
* UMR 7503 CNRS-INPL-INRIA-Nancy2-UHP
} 
has to follow, and deductive as well as some computing abilities which empower the user. The proof system may then be used to backup automated theorem provers such as Simplify, Harvey, Zenon or implemented in interactive proof assistants like Coq, Isabelle, PVS or HOL that work as a framework for the proof engineering process. In this context, a proof is usually constructed with respect to a theory (a set of axioms) which is plugged into the deduction process. Indeed the deductive power of a deduction system comes from its association with a theory. In frameworks such as first-order natural deduction or sequent calculus, the use of some theory is always uniform since the proofs only express atomic steps which correspond to decompositions of logical connectives. Higher-level notions such as sets, set inclusion, natural numbers or addition have to be encoded in the first-order language and handled through these atomic inference steps, leading often to long, hardly-readable and redundant "assembly like" proofs.

Several paradigms propose ad hoc systems for specific theories. Let us cite Huang's Assertion level [1] mainly motivated by the presentation of machine found proofs in natural language. Another approach proposed by Negri and von Plato in [2] is closely related to the handling of axioms which we will use here. They express first-order axioms through so-called non-logical rules. However these inference rules only act on the left-hand side of sequents. Consequently they can only poorly interact with an elimination of cuts. Our approach is much closer to Definitional Reflection [3], which is extended with induction in [4]. Indeed Definitional Reflection adds left and right introduction rules to an intuitionistic sequent calculus in order to reflect some definitional clause which is in turn a special axiom, corresponding to what we will call proposition rewrite rules. In addition, cut-elimination results are proven for these logics with definitions and induction. However working in classical sequent calculus will allow us to deal with more general definitions. Besides our main concern in this paper will not be the normalisation of proofs, even if our approach is provided in [5] with a proofterm language and a cut-elimination procedure which is proven to be strongly normalising under appropriate assumptions.

The approach we are about to study in this paper is related to Focusing introduced by Andreoli in [6] which was meant to remove irrelevant choices in backward reasoning for sequent calculus: Indeed syntactically different proofs can still be identical up to some permutations or simplifications of the applications of the inference rules. Our point of view is that usually when one wishes to reason with an axiom (such as one used in the definition of natural numbers), in sequent calculus for instance, it is necessary to destruct the logical connectives of the axiom. These steps are typically always identical, at least up to permutations or simplifications of the applications of the inference rules. Superdeduction, introduced in [5], is an adaptation of the focusing paradigm especially designed to reason with axiom: It allows to use the deductive part of some theory to enrich the deductive system, obtaining thus a custom-made system devoted to the theory. However there is still a gap between focusing and superdeduction, which we propose to fill in this paper. Superdeduction was initially founded on the work of Benjamin Wack in [7] on supernatural deduction which was introduced 
in order to provide a logical interpretation to the rewriting calculus $[8,9]$. Based on Urban's work on the classical sequent calculus [10], a proof-term language for superdeduction is proposed in [5] along with a cut-elimination procedure whose normalisation is proved in [11] under appropriate hypotheses.

In this paper superdeduction is combined with a dual approach which is also designed to structure the use of some first-order theory: Deduction modulo, which was introduced ten years ago by Dowek, Hardin and Kirchner [12]. It is a paradigm which allows one to transform any formula at any point of some deduction process. Since the transformation is not explicit in the constructed proof, it has to be redone during proofchecking and therefore cannot be more than computation. For instance it is commonly defined as a congruence on formulæ which is constructed from a convergent rewrite system [13]. Since the rewrite system is convergent, verifying some formula transformation is decidable. Hence deduction modulo allows to make use of the computational part of a theory $\mathcal{T}$ for true computations modulo which deduction is performed. Deduction modulo leads to interesting automated theorem proving procedures like ENAR [12] or TaMeD [14]. Another line of work is to formalize theories of interest, such as Heyting arithmetic [15,16], Zermelo's set theory [17] or higher-order logics $[18,19,20]$, using rewrite systems so that they can be used in deduction modulo. Finally, besides simplicity, deduction modulo admits unbounded proof size speed-up [21].

In this work, these two approaches are combined in the following way, as it was already put forward in [5]. Instead of constructing a proof of $\mathcal{T} h \vdash \Delta$ in natural deduction or sequent calculus using the theory $\mathcal{T}$ uniformly, we propose to split $\mathcal{T} h$ in three parts $\Gamma \cup \mathcal{T} h_{1} \cup \mathcal{T} h_{2}$. While $\Gamma$ will still be used as a context to our deductions, $\mathcal{T} h_{1}$ will represent computational axioms and will be handled by the deduction modulo paradigm, and $\mathcal{T} h_{2}$ will represent reasoning axioms and therefore will be handled by the superdeduction paradigm. We obtain then a custom deduction system in which we prove a sequent $\Gamma \vdash_{\equiv_{T h_{1}}^{+T h_{2}}}^{+ \text {. }}$

As already explained in [5], superdeduction modulo may be used as an innovative foundation for new proof assistants. First it allows to naturally encode custom reasoning and computational schemes, such as induction over natural numbers and addition. Furthermore it suggests a convenient representation of proofs especially adapted to human interaction and in contrast with the proofs that are typically constructed with existing proof-assistants such as Coq or Isabelle. These usually consist in tactics or proof-terms which are bound to convince the user of the correctness of the proof (through a typechecking process) but not actually explain it. Indeed the main steps are flooded with a multitude of usually not explicit and often straightforward logical arguments caused by both the underlying calculus and the presence of purely computational parts. While deduction modulo addresses the issue of computational parts, superdeduction eliminates trivial logical arguments in a proof. Moreover the expressiveness of superdeduction modulo is promising. For instance an encoding of Pure Type Systems in (a weak version of) superdeduction modulo is shown in [22].

Using awkward theories in deduction modulo or superdeduction can break cut-elimination (admissibility of the cut-rule) and strong normalisation (of a 
cut-elimination procedure), even for consistent theories. However several criteria exists for theories which do not threaten cut-elimination or strong normalisation when used in deduction modulo: Criteria have been developed for strong normalisation, using reducibility candidates [23] leading to the notion of truth values algebras and superconsistency $[24,25]$, and for cut-elimination using semantic methods $[26,27]$ or abstract completion [28]. One of our aims in this paper is to relate superdeduction modulo and sheer deduction modulo by proving (under appropriate hypotheses) that cut-elimination in one system is equivalent to cut-elimination in the other. Indeed we prove that any cut-free proof of

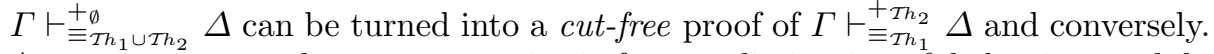
As a consequence, the numerous criteria for cut-elimination of deduction modulo also hold for superdeduction modulo. Finally this cut-elimination result allows us to write a sound and complete tableaux method for superdeduction modulo.

The definition of superdeduction modulo is written in Section 2. In this context, our contributions are the following: First we give a presentation of superdeduction modulo through a focusing system, which consequently leads to a clear comparison of superdeduction with the focusing approach (Section 3). Then we prove a focalization theorem for superdeduction modulo, which states that a cut-free proof in superdeduction modulo can be translated into a cut-free proof in raw deduction modulo (the converse being obvious), and which leads to criteria for theories which can extend deduction through the superdeduction modulo paradigm without endangering cut-elimination (Section 4); Finally we design a tableau method for superdeduction modulo which is sound and complete, provided cut-elimination holds (Section 5). Detailed proofs of lemmas and theorems are written in Appendix A.

This article is a long version of a paper submitted to LPAR 2008.

\section{Superdeduction Modulo}

In this section we define superdeduction modulo which is the combination of deduction modulo and superdeduction. Let us recall the classical sequent calculus LK which contains the deduction core of our superdeduction modulo systems.

$$
\begin{aligned}
& \mathrm{Ax} \overline{\Gamma, \varphi \vdash \varphi, \Delta} \perp_{L} \overline{\Gamma, \perp \vdash \Delta} \top_{R} \overline{\Gamma \vdash \top, \Delta} \\
& \wedge_{L} \frac{\Gamma, \varphi_{1}, \varphi_{2} \vdash \Delta}{\Gamma, \varphi_{1} \wedge \varphi_{2} \vdash \Delta} \wedge_{R} \frac{\Gamma \vdash \varphi_{1}, \Delta \quad \Gamma \vdash \varphi_{2}, \Delta}{\Gamma \vdash \varphi_{1} \wedge \varphi_{2}, \Delta} \vee_{L} \frac{\Gamma, \varphi_{1} \vdash \Delta \quad \Gamma, \varphi_{2} \vdash \Delta}{\Gamma, \varphi_{1} \vee \varphi_{2} \vdash \Delta} \\
& \Rightarrow_{R} \frac{\Gamma, \varphi_{1} \vdash \varphi_{2}, \Delta}{\Gamma \vdash \varphi_{1} \Rightarrow \varphi_{2}, \Delta} \quad \Rightarrow_{L} \frac{\Gamma \vdash \varphi_{1}, \Delta \quad \Gamma, \varphi_{2} \vdash \Delta}{\Gamma, \varphi_{1} \Rightarrow \varphi_{2} \vdash \Delta} \vee_{R} \frac{\Gamma \vdash \varphi_{1}, \varphi_{2}, \Delta}{\Gamma \vdash \varphi_{1} \vee \varphi_{2}, \Delta} \\
& \forall_{R} \frac{\Gamma \vdash \varphi, \Delta}{\Gamma \vdash \forall x . \varphi, \Delta} x \notin \mathcal{F} \mathcal{V}(\Gamma, \Delta) \quad \forall_{L} \frac{\Gamma, \varphi[t / x] \vdash \Delta}{\Gamma, \forall x . \varphi \vdash \Delta} \exists_{L} \frac{\Gamma, \varphi \vdash \Delta}{\Gamma, \exists x . \varphi \vdash \Delta} x \notin \mathcal{F V}(\Gamma, \Delta) \\
& \mathrm{C}_{R} \frac{\Gamma \vdash \varphi, \varphi, \Delta}{\Gamma \vdash \varphi, \Delta} \mathrm{C}_{L} \frac{\Gamma, \varphi, \varphi \vdash \Delta}{\Gamma, \varphi \vdash \Delta} \quad \text { Cut } \frac{\Gamma \vdash \varphi, \Delta \quad \Gamma, \varphi \vdash \Delta}{\Gamma \vdash \Delta} \exists_{R} \frac{\Gamma \vdash \varphi[t / x], \Delta}{\Gamma \vdash \exists x \cdot \varphi, \Delta}
\end{aligned}
$$


A term rewrite rule rewrites first-order terms into first-order terms and a proposition rewrite rule rewrites an atomic proposition into an arbitrary formula. For instance plus(zero, $x) \rightarrow x$ is a term rewrite rule while $a \subseteq b \rightarrow \forall x . x \in$ $a \Rightarrow x \in b$ is a proposition rewrite rule. We will consider two sets of rewrite rules: $\mathcal{T} h_{1}$ contains both term and proposition rewrite rules ; $\mathcal{T} h_{2}$ is a set of proposition rewrite rules. As $\mathcal{T} h_{1}$ will be used to extend the deduction system through deduction modulo, our intuition is that it contains the computational axioms. Dually as $\mathcal{T} h_{2}$ will be used to enrich the deduction system through superdeduction, our intuition is that it contains the deductive axioms. We suppose that each rewrite rule $P \rightarrow \varphi$ of $\mathcal{T h}_{2}$ is associated with some name $\mathrm{R}$ then denoted $\mathrm{R}: P \rightarrow \varphi$. For $i \in\{1,2\}$, the one-step rewrite reduction associated with $\mathcal{T h}_{i}$ is denoted $\rightarrow_{i}$. The reflexive and transitive closure of $\rightarrow_{i}$ is denoted $\rightarrow_{i}^{*}$. The symmetric, reflexive and transitive closure of $\rightarrow_{i}$ is denoted $\equiv_{i}$. The notations $\rightarrow_{1,2}, \rightarrow_{1,2}^{*}$, and $\equiv_{1,2}$ are used for $\mathcal{T} h_{1} \cup \mathcal{T} h_{2}$. The first-order axiom associated with a proposition rewrite rule $P \rightarrow \varphi$ is $\forall \bar{x} .(P \Leftrightarrow \varphi)$, where $\bar{x}$ represents the free variables of $P$ and $\varphi$, denoted $\mathcal{F} \mathcal{V}(P, \varphi)$. The first-order axiom associated with a term rewrite rule $l \rightarrow r$ is $\forall \bar{x}$. $(l=r)$, where $\bar{x}$ represents the free variables of $l$ and $r$. This way of representing a term rewrite rule as a first-order axiom supposes that the logic contains an equality symbol. If it does not, one may add this predicate and the corresponding axioms in a conservative way as detailed in [29]. An other way to proceed with the term rewrite rules of $\mathcal{T} h_{1}$ is to use axioms of the form $\forall \bar{x}$. $P \Leftrightarrow Q$ for all $P \equiv_{1} Q$. When writing $\vdash_{\equiv^{T} h_{1}}^{+T h_{2}}, \mathcal{T} h_{1}$ and $\mathcal{T} h_{2}$ will represent the rewrite rules, and when writing $\mathcal{T} h_{1} \vdash$ or $\mathcal{T} h_{2} \vdash$, they will represent the first-order axioms. For some deduction system $\vdash_{*}^{\star}$, we will just write $\Gamma \vdash_{*}^{\star} \Delta$ instead of the sentence there is a proof of $\Gamma \vdash_{*}^{\star} \Delta$. The sequents in the corresponding cut-free deduction system will be denoted $\Gamma \vdash_{*}^{c f \star} \Delta$.

Superdeduction stands for the addition to LK of new superdeduction inference rules that are computed from $\mathcal{T} h_{2}$ in the following way.

Definition 1 (Superdeduction rules computation [5]) Let Calc be the set of inference rules formed of $\mathrm{Ax}, \perp_{L}, \top_{R}, \vee_{L}, \vee_{R}, \wedge_{L}, \wedge_{R}, \Rightarrow_{L}, \Rightarrow_{R}, \forall_{L}, \forall_{R}$, $\exists_{L}, \exists_{R}, \top_{L} \frac{\Gamma \vdash \Delta}{\Gamma, \top \vdash \Delta}$ and $\perp_{R} \frac{\Gamma \vdash \Delta}{\Gamma \vdash \perp, \Delta}$. Let us suppose $\mathrm{R}: P \rightarrow \varphi \in \mathcal{T h}_{2}$. To get the right (resp. left) rule associated with $\mathrm{R}$, apply bottom-up the rules of Calc to the sequent $\Gamma \vdash \varphi, \Delta$ (resp. $\Gamma, \varphi \vdash \Delta$ ) until no connective of $\varphi$ remain, collect the premises and side conditions, and finally replace $\varphi$ by $P$ in the conclusion.

For instance, the rules associated with $\subseteq_{\text {def }}: a \subseteq b \rightarrow \forall x . x \in a \Rightarrow x \in b$ are

$$
\subseteq_{\operatorname{def}_{R}} \frac{\Gamma, x \in a \vdash x \in b, \Delta}{\Gamma \vdash a \subseteq b, \Delta} x \notin \mathcal{F} \mathcal{V}(\Gamma, \Delta) \quad \subseteq_{\operatorname{def}_{L}} \frac{\Gamma, t \in b \vdash \Delta \quad \Gamma \vdash t \in a, \Delta}{\Gamma, a \subseteq b \vdash \Delta}
$$

Since the propositional rules of Calc commute with any other rules, they may be applied in any order to reach axioms. However the application order of rules concerning quantifiers can be significant. Even if it will not affect the resulting premises, the side condition may differ: Decomposing $P \rightarrow(\exists x . A(x)) \Rightarrow$ $(\exists x . B(x))$ on the right can lead to 


$$
\frac{\Gamma, A(x) \vdash B(t), \Delta}{\Gamma \vdash P, \Delta} x \notin \mathcal{F} \mathcal{V}(\Gamma, \Delta) \quad \text { or } \quad \frac{\Gamma, A(x) \vdash B(t), \Delta}{\Gamma \vdash P, \Delta} x \notin \mathcal{F} \mathcal{V}(\Gamma, \Delta, t)
$$

depending on which existential quantifier is decomposed first. Although both rules are sound, only the first is complete. Therefore when computing superdeduction rules, we give $\exists_{L}$ and $\forall_{R}$ a higher priority than the other rules, consequently ensuring that we always obtain the weakest side condition.

Definition 2 (Superdeduction modulo) The superdeduction modulo system associated with $\left(\mathcal{T} h_{1}, \mathcal{T h}_{2}\right)$ is formed of the rules of $L K$ and the rules built upon $\mathrm{Th}_{2}$, where each first-order term or proposition is considered modulo rewriting

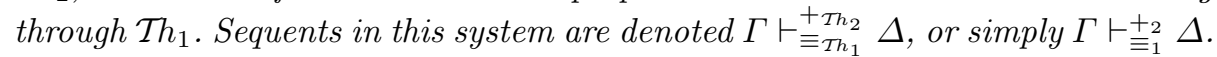

If $\mathcal{T h} h_{2}$ is empty, then we are in raw deduction modulo and sequents are denoted $\Gamma \vdash_{\equiv_{1}} \Delta$. If $\mathcal{T} h_{1}$ is empty, then we are in raw superdeduction and sequents are denoted $\Gamma \vdash^{+2} \Delta$. There are two equivalent ways to present the use of inference rules where propositions and first-order terms are considered modulo rewriting through $\mathcal{T} h_{1}$ : One can use new inference rules such as

$$
\equiv_{1 R} \frac{\Gamma \vdash \psi, \Delta}{\Gamma \vdash \varphi, \Delta} \psi \equiv_{1} \varphi \quad \equiv_{1 L} \frac{\Gamma, \psi \vdash \Delta}{\Gamma, \varphi \vdash \Delta} \psi \equiv_{1} \varphi
$$

or one can replace the inference rules of the system by rules such as

$$
\Rightarrow_{R} \frac{\Gamma, \varphi_{1} \vdash \varphi_{2}, \Delta}{\Gamma \vdash \varphi, \Delta} \varphi \equiv_{1} \varphi_{1} \Rightarrow \varphi_{2} \quad \text { Cut } \frac{\Gamma \vdash \varphi, \Delta \quad \Gamma, \psi \vdash \Delta}{\Gamma \vdash \Delta} \varphi \equiv_{1} \psi \quad \ldots
$$

For example in this latter presentation, the rules associated with $\subseteq_{\text {def }}$ become

$\subseteq_{\operatorname{def} R} \frac{\Gamma, x \in a \vdash x \in b, \Delta}{\Gamma \vdash \varphi, \Delta}\left\{\begin{array}{l}x \notin \mathcal{F} \mathcal{V}(\Gamma, \Delta) \\ \varphi \equiv_{1} a \subseteq b\end{array} \subseteq_{\operatorname{def} L} \frac{\Gamma, t \in b \vdash \Delta \Gamma \vdash t \in a, \Delta}{\Gamma, \varphi \vdash \Delta} \varphi \equiv_{1} a \subseteq b\right.$

Let us recall that we allow $\mathcal{T} h_{1}$ to contain proposition rewrite rules (rewriting on formulæ). This can lead to odd situations: For instance a conjunction $\varphi_{1} \wedge \varphi_{2}$ may be congruent to a disjunction $\psi_{1} \vee \psi_{2}$, and then one can perform a cut between a decomposition of the first on the left and a decomposition of the second on the right.

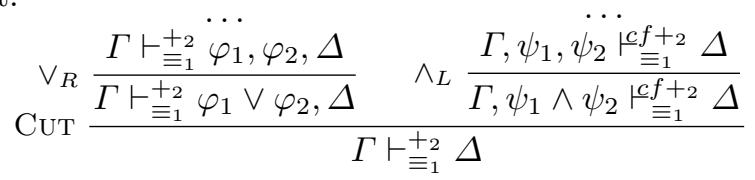

Obviously there is no way to eliminate this cut a priori, especially when $\varphi_{i} \equiv_{1} \psi_{j}$ for all $i, j \in\{1,2\}$. In order to avoid this kind of awkward situation, deduction modulo is usually only considered for confluent rewrite systems: Since $\mathcal{T} h_{1}$ only rewrites first-order terms and atomic predicates, if $\varphi_{1} \wedge \varphi_{2} \equiv_{1} \psi_{1} \vee \psi_{2}$ and if $\mathcal{T} h_{1}$ is confluent, then the common reduct of $\varphi_{1} \wedge \varphi_{2}$ and $\psi_{1} \vee \psi_{2}$ is both a conjunction and a disjunction, which is contradictory. Consequently we will always suppose that $\mathcal{T} h_{1}$ is confluent besides of only rewriting atoms.

It is interesting to notice that our superdeduction modulo systems are combination of superdeduction and deduction modulo in the following order: first 
the superdeduction inference rules are computed, then they are used modulo rewriting. Therefore deduction modulo does not interfere with the computation of the superdeduction rules, but only with their use. However we could have chosen to compute these new inference rules by already using deduction modulo. This computation is deterministic if and only if $\mathcal{T} h_{1}$ is confluent: Indeed in this case, rewriting through $\mathcal{T} h_{1}$ cannot erase the logical connectives of some formula (since it only rewrites atomic predicates) and cannot spawn distinct logical connectives at the same position of the formula (since it is confluent). Besides the computation is terminating if and only if $\mathcal{T} h_{1}$ is terminating.

The first properties of superdeduction modulo systems that we will prove are its soundness (in the present section) and completeness (in the following section) w.r.t. classical predicate logic. The first is a straightforward consequence of the soundness of deduction modulo proven in [12]:

Theorem 1 ([12]) $\Gamma \vdash_{\equiv_{1}} \Delta$ if and only if $\mathcal{T} h_{1}, \Gamma \vdash \Delta$.

The soundness of superdeduction modulo is then a direct consequence of the following lemma.

Lemma 1 (From $\vdash_{\equiv_{1}}^{+2}$ to $\vdash_{\equiv_{1,2}}$ )

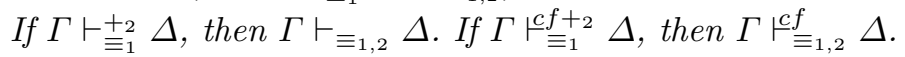

Proof. By definition of the superdeduction rules for $P \rightarrow \varphi \in \mathcal{T h}_{2}$, they can be replaced by several rules of Calc followed by a conversion step $\varphi \equiv_{2} P$. The proof is detailed in Appendix A.

Theorem 2 (Soundness of $\vdash_{\equiv_{1}^{+2}}^{+}$) If $\Gamma \vdash_{\equiv_{1}}^{+2} \Delta$, then $\mathcal{T h}, \mathcal{T} h_{2}, \Gamma \vdash \Delta$.

Proof. Using Lemma 1, the proof of $\Gamma \vdash_{\equiv_{1}^{+2}}^{+} \Delta$ is translated into a proof of $\Gamma \vdash_{\equiv_{1,2}}$ $\Delta$, then by soundness of deduction modulo into a proof of $\mathcal{T} h_{1}, \mathcal{T} h_{2}, \Gamma \vdash \Delta$.

Let us now demonstrate the use of superdeduction modulo through an example. Deduction modulo is convenient for representing the computational part of some theory. For instance one can define the addition using $0+y \rightarrow y$ and $S(x)+y \rightarrow S(x+y)$, and the multiplication using $0 * x \rightarrow 0$ and $S(x) * y \rightarrow$ $y+(y * x)$. Let us also define $\operatorname{sum}(x)=\sum_{k=0}^{x-1}(2 * k+1)$ with the rules $\operatorname{sum}(0) \rightarrow 0$ and $\operatorname{sum}(S(x)) \rightarrow S(x+(x+\operatorname{sum}(x)))$. We obtain a convergent rewrite system, which is suitable for representing computation in deduction modulo. Superdeduction is convenient for representing the deductive part of a theory. Let us define natural numbers (and induction) using the rules $\mathbb{N}(n) \rightarrow \forall P .0 \in P \Rightarrow \mathfrak{H}(P) \Rightarrow n \in P$ and $\mathfrak{H}(P) \rightarrow \forall k . k \in P \Rightarrow S(k) \in P$. Let us also define Leibniz's equality with the rule $x=y \rightarrow \forall P . x \in P \Rightarrow y \in P$. In the three latter rules, we wrote $x \in P$ instead of $P(x)$, for some formula $P$. This is allowed if we use (in $\mathcal{T} h_{1}$ ) the axiom $x \in \tilde{P} \rightarrow P(x)$ coming from the theory of classes, where $\tilde{P}$ denotes fresh constants associated with each formula $P$. The new inference rules for natural 
numbers and equality are then

$$
\begin{aligned}
& \mathbb{N}_{R} \frac{\Gamma, 0 \in P, \mathfrak{H}(P) \vdash_{\equiv}^{+2} n \in P, \Delta}{\Gamma \vdash_{\equiv_{1}}^{+2} \mathbb{N}(n), \Delta} P \notin \mathcal{F} \mathcal{V}(\Gamma, \Delta) \quad \mathfrak{H}_{L} \frac{\Gamma \vdash_{\equiv_{1}^{+2}}^{++_{2}} m \in P, \Delta \quad \Gamma, S(m) \in P \vdash_{\equiv_{1}}^{+2} \Delta}{\Gamma, \mathfrak{H}(P) \vdash_{\equiv_{1}}^{+2} \Delta}
\end{aligned}
$$

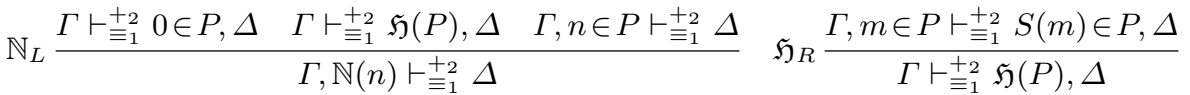

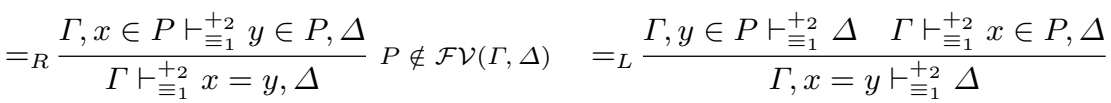

Besides, the system is considered modulo the rules for addition, multiplication and $\operatorname{sum}(x)$. Then one can easily prove in the system that $\mathbb{N}(n)$ implies $\sum_{k=0}^{n-1}(2 * k+1)=n^{2}$. The proof is

$$
\begin{aligned}
& \operatorname{Ax}(S(m+(m+\operatorname{sum}(m)))=S(m+(m+(m * m)))) \\
& ={ }_{R} \frac{\operatorname{Ax}(S(m+(m+\operatorname{sum}(m))) \in A)}{\vdash_{\equiv_{1}}^{+2} S(m+(m+\operatorname{sum}(m)))=S(m+(m+\operatorname{sum}(m)))} \quad \vdots \\
& { }_{\mathfrak{H}_{R}} \overline{\operatorname{sum}(m)=m * m \vdash_{\equiv_{1}^{+2}}^{+2} S(m+(m+\operatorname{sum}(m)))=S(m+(m+(m * m)))}
\end{aligned}
$$

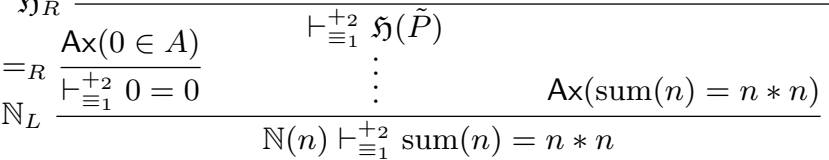

where $\tilde{P}$ is the first-order constant associated with the formula $\operatorname{sum}(m)=m * m$

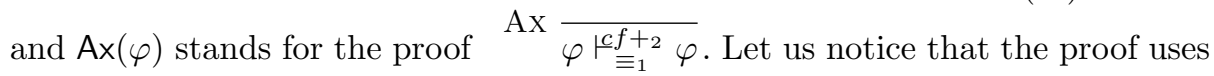
only superdeduction rules and Ax rules.

\section{$3 \quad$ Superdeduction and focusing systems}

The aim of this section is to explore how focusing and superdeduction are related. For example the distinction between the two kinds of sequents that focusing introduces, namely unfocused and focused sequents, is very similar to the distinction between sequents used in the superdeduction toplevel and (meta)sequents used during the superdeduction inference rules computation: In this latter case, a specific formula $\varphi$ appearing in a proposition rewrite rule $P \rightarrow \varphi$ replaces $P$ and is then focused until the complete decomposition of its connectives.

Let us propose an extension of classical sequent calculus based on this distinction between focused and unfocused sequents, but more general than both focusing and superdeduction in the sense that both of them are instances of this extension. Starting from the classical sequent calculus LK (as written in Section 2 ), we add the following rules. 


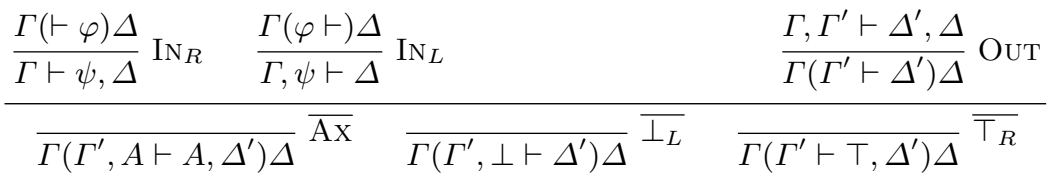

$$
\begin{aligned}
& \frac{\Gamma\left(\Gamma^{\prime} \vdash \Delta^{\prime}\right) \Delta}{\Gamma\left(\Gamma^{\prime} \vdash \perp, \Delta^{\prime}\right) \Delta} \bar{\perp}_{R} \quad \frac{\Gamma\left(\Gamma^{\prime} \vdash \Delta^{\prime}\right) \Delta}{\Gamma\left(\Gamma^{\prime}, \top \vdash \Delta^{\prime}\right) \Delta} \overline{\top_{L}} \\
& \frac{\Gamma\left(\Gamma^{\prime} \vdash \varphi_{1}, \Delta^{\prime}\right) \Delta \quad \Gamma\left(\Gamma^{\prime} \vdash \varphi_{2}, \Delta^{\prime}\right) \Delta}{\Gamma\left(\Gamma^{\prime} \vdash \varphi_{1} \wedge \varphi_{2}, \Delta^{\prime}\right) \Delta} \overline{\wedge_{R}} \quad \frac{\Gamma\left(\Gamma^{\prime}, \varphi_{1}, \varphi_{2} \vdash \Delta^{\prime}\right) \Delta}{\Gamma\left(\Gamma^{\prime}, \varphi_{1} \wedge \varphi_{2} \vdash \Delta^{\prime}\right) \Delta} \overline{\wedge_{L}} \\
& \frac{\Gamma\left(\Gamma^{\prime} \vdash \varphi_{1}, \varphi_{2}, \Delta^{\prime}\right) \Delta}{\Gamma\left(\Gamma^{\prime} \vdash \varphi_{1} \vee \varphi_{2}, \Delta^{\prime}\right) \Delta} \nabla_{R} \quad \frac{\Gamma\left(\Gamma^{\prime}, \varphi_{1} \vdash \Delta^{\prime}\right) \Delta \quad \Gamma\left(\Gamma^{\prime}, \varphi_{2} \vdash \Delta^{\prime}\right) \Delta}{\Gamma\left(\Gamma^{\prime}, \varphi_{1} \vee \varphi_{2} \vdash \Delta^{\prime}\right) \Delta} \nabla_{L} \\
& \frac{\Gamma\left(\Gamma^{\prime}, \varphi_{2} \vdash \Delta^{\prime}\right) \Delta \quad \Gamma\left(\Gamma^{\prime} \vdash \varphi_{1}, \Delta^{\prime}\right) \Delta}{\Gamma\left(\Gamma^{\prime}, \varphi_{1} \Rightarrow \varphi_{2} \vdash \Delta^{\prime}\right) \Delta} \Rightarrow_{R} \quad \frac{\Gamma\left(\Gamma^{\prime}, \varphi_{1} \vdash \varphi_{2}, \Delta^{\prime}\right) \Delta}{\Gamma\left(\Gamma^{\prime} \vdash \varphi_{1} \Rightarrow \varphi_{2}, \Delta^{\prime}\right) \Delta} \Rightarrow_{L} \\
& \bar{\forall}_{R} \frac{\Gamma\left(\Gamma^{\prime} \vdash \varphi, \Delta^{\prime}\right) \Delta}{\Gamma\left(\Gamma^{\prime} \vdash \forall x . \varphi, \Delta^{\prime}\right) \Delta} x \notin \mathcal{F} \mathcal{V}\left(\Gamma, \Gamma^{\prime}, \Delta, \Delta^{\prime}\right) \quad \bar{\forall}_{L} \frac{\Gamma\left(\Gamma^{\prime}, \varphi[t / x] \vdash \Delta^{\prime}\right) \Delta}{\Gamma\left(\Gamma^{\prime}, \forall x . \varphi \vdash \Delta^{\prime}\right) \Delta} \\
& \bar{\exists}_{R} \frac{\Gamma\left(\Gamma^{\prime} \vdash \varphi[t / x], \Delta^{\prime}\right) \Delta}{\Gamma\left(\Gamma^{\prime} \vdash \exists x . \varphi, \Delta^{\prime}\right) \Delta} \quad \overline{\exists_{L}} \frac{\Gamma\left(\Gamma, \varphi \vdash \Delta^{\prime}\right) \Delta}{\Gamma\left(\Gamma, \exists x . \varphi \vdash \Delta^{\prime}\right) \Delta} x \notin \mathcal{F} \mathcal{V}\left(\Gamma, \Gamma^{\prime}, \Delta, \Delta^{\prime}\right)
\end{aligned}
$$

The system features then two kinds of sequents: Unfocused sequents denoted $\Gamma \vdash \Delta$ and focused sequents denoted $\Gamma\left(\Gamma^{\prime} \vdash \Delta^{\prime}\right) \Delta$. Unfocused sequents are handled by the rules of classical sequent calculus; focused sequents are handled by the overlined rules; entering a focusing sequence is handled by rules $\mathrm{IN}_{R}$ and $\mathrm{IN}_{L}$; finally leaving a focusing sequence is handled by rule OUT. In addition, the obtained sequent calculus has the three following parameters: A condition $\mathcal{C}_{\text {IN }}$ which is enforced when applying the rules $\mathrm{IN}_{R}$ and $\mathrm{IN}_{L}$, a condition $\mathcal{C}_{\text {Out }}$ which is enforced when applying the rule OUT, a condition $\mathcal{C}_{\text {Focus }}$ which is enforced when applying any of the overlined rules and a condition $\mathcal{C}_{\text {Unfocus }}$ which is enforced when applying any of the raw LK rules. Therefore the resulting deduction system is denoted $\operatorname{LK}\left(\mathcal{C}_{\text {IN }}, \mathcal{C}_{\text {OUt }}, \mathcal{C}_{\text {Focus }}, \mathcal{C}_{\text {Unfocus }}\right) . \mathcal{C}_{\text {IN }}$ and $\mathcal{C}_{\text {OUt }}$ are meant to respectively control the bottom-up entrance and exit of focusing phases; $\mathcal{C}_{\text {Focus }}$ and $\mathcal{C}_{\text {Unfocus }}$ are meant to respectively control the deduction inside of focused and unfocused phases. For instance if $\mathcal{C}_{\mathcal{T} h_{1}}$ is the condition that forces $\varphi \equiv_{1} \psi$ in the rules $\operatorname{IN}_{R}$ and $\mathrm{IN}_{L}$, then $\operatorname{LK}\left(\mathcal{C}_{\mathcal{T} h_{1}}\right.$, true, false, true) is equivalent to deduction modulo $\mathcal{T} h_{1}$ : The overlined rules are simply discarded, and the $\mathrm{IN}_{R}$ and $\mathrm{IN}_{L}$ rules are turned into conversion rules for $\mathcal{T} h_{1}$. Both focusing proofs and superdeduction can also be formulated as instances of $\operatorname{LK}\left(\mathcal{C}_{\text {IN }}, \mathcal{C}_{\text {OUt }}, \mathcal{C}_{\text {Focus }}, \mathcal{C}_{\text {Unfocus }}\right)$.

Focusing. We will say that a (focused or unfocused) sequent is positive if the head connectives of its non-atomic formulæ are $\forall$ on the left and $\exists$ on the right. Then one can define the following focusing system for LK: $\mathcal{F}_{+}$is the condition which restricts the conclusion of the applied inference rule to be a positive sequent. $\mathcal{F}_{-}$is the condition which restricts the conclusion of the applied inference rule not to be a positive sequent. Then $\operatorname{LK}\left(\mathcal{F}_{+} \wedge \varphi=\psi, \mathcal{F}_{-}, \mathcal{F}_{+}, \mathcal{F}_{-}\right)$is a focusing system for LK. It is quite different from the focusing system LKF presented in [30], in particular regarding our asynchronous treatment of propositional connectives. However both systems only allow to focus on a single formula. It is syntactically ensured in LKF, while ensured by $\mathcal{F}_{+}$together with the shape of 
$\overline{\exists_{R}}$ and $\overline{\forall_{L}}$ (the only overlined rules allowed by $\left.\mathcal{F}_{+}\right)$in $\operatorname{LK}\left(\mathcal{F}_{+}, \mathcal{F}_{-}, \mathcal{F}_{+}, \mathcal{F}_{-}\right.$). Focused sequents which are denoted $\mapsto[\Theta], P$ in [30], are denoted $(\vdash P) \Theta$ here.

Superdeduction. The explicit superdeduction system associated with $\mathcal{T h}_{2}$ is defined as $\operatorname{LK}\left(\mathcal{C}_{\mathcal{T h}_{2}}, \mathcal{C}_{\text {atoms }}\right.$, true, true) where $\mathcal{C}_{\mathcal{T h}_{2}}$ is the condition (for the application of rules $\mathrm{IN}_{R}$ and $\mathrm{IN}_{L}$ ) which states that $\psi \rightarrow \varphi$ is a rule of $\mathcal{T} h_{2}$ and $\mathcal{C}_{\text {atoms }}$ is the condition which restricts the focused part of the conclusion of the rule OUt to contain only atoms. $\operatorname{LK}\left(\mathcal{C}_{\mathcal{T h}}, \mathcal{C}_{\text {atoms }}\right.$, true, true $)$ is equivalent to the superdeduction system associated with $\mathcal{T} h_{2}$, since each focused phase exactly corresponds with the computation of some superdeduction inference rule.

On the one hand, $\operatorname{LK}\left(\mathcal{C}_{\text {IN }}, \mathcal{C}_{\text {OUT }}, \mathcal{C}_{\text {Focus }}, \mathcal{C}_{\text {Unfocus }}\right)$, as the supremum of focusing and superdeduction, makes clear the similarities between these two approaches:

- Focused and unfocused sequents are syntactically identified.

- Focused sequents focus on a special part of the sequent.

- The system features three kinds of inferences which either handle focused sequents, handle unfocused sequents or controls the enter/exit of the focus.

On the other hand, it also underlines the dissimilarities:

- Focused sequents usually focus on a single formula in the focusing approach, but they may focus on several in the superdeduction approach.

- Focusing phases of superdeduction contain an unfolding step (IN rules) besides purely logical steps.

- The focusing approach divides logical connectives into positive and negative connectives. Usually, no connective is both positive and negative, and the De Morgan dual of a positive connective is negative and conversely. On the contrary, superdeduction locally divides connectives into toplevel connectives (decomposed in unfocused phases) and connectives appearing in axioms of $\mathcal{T h}_{2}$, but a conjunction, for instance, can appear as a toplevel connective somewhere and in the explicit decomposition of a superdeduction axiom.

- In the focusing approach, $\mathcal{F}_{+}$and $\mathcal{F}_{-}$state that the focusing phase is entered (bottom-up) only when no negative connective remains (the latest possible), and is left as soon as a negative connective is found (the soonest possible). This aspect plays an important role in the completeness of the focusing approach. In the superdeduction approach, $\mathcal{C}_{\mathcal{T h}}$ and $\mathcal{C}_{\text {atoms }}$ state that the focusing phase is entered anytime a superdeduction axiom is unfolded, and is left only when no connective of the axiom remains (the latest).

More generally, let us stress the fact in the superdeduction approach, the focused phases are directed by axioms of $\mathcal{T h}_{2}$ (therefore axiom directed focusing). In particular these axioms direct when the focusing phases can be entered and exited. They also direct which connectives will be decomposed during a single focusing phase. Consequently one can propose syntactical criteria on $\mathcal{T h}_{2}$ to ensure completeness or cut-elimination of the system. Completeness is the subject of the remaining of the section. Cut-elimination will be handled in Section 4 . But let us introduce some terminology first. 
Definition 3 (Polarity of a subformula) The polarity $\operatorname{pol}_{\varphi}(\psi)$ of $\psi$ in $\varphi$ where $\psi$ is an occurrence of a subformula of $\varphi$ is defined as true if $\varphi=\psi$; $\operatorname{pol}_{\varphi_{i}}(\psi) \quad$ if $\varphi=\varphi_{1} \wedge \varphi_{2}$ or $\varphi_{1} \vee \varphi_{2}$ or $\forall x . \varphi_{1}$ or $\exists x . \varphi_{1}$, and $\psi$ occurs in $\varphi_{i}$; $\neg \operatorname{pol}_{\varphi_{1}}(\psi)$ if $\varphi=\varphi_{1} \Rightarrow \varphi_{2}$ and if $\psi$ occurs in $\varphi_{1}$; $\operatorname{pol}_{\varphi_{2}}(\psi) \quad$ if $\varphi=\varphi_{1} \Rightarrow \varphi_{2}$ and if $\psi$ occurs in $\varphi_{2}$.

There are four cases for which two steps of LK cannot be permuted:

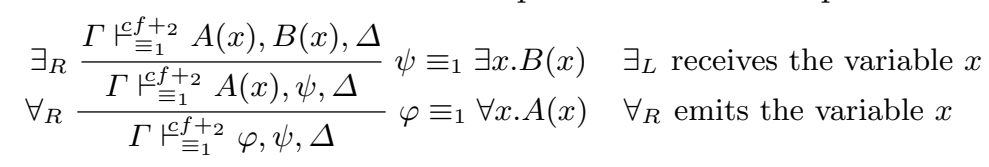

and similarly where $\forall_{R}$ is replaced by $\exists_{L}$ and/or $\exists_{R}$ is replaced by $\forall_{L}$. It leads to the following definition of positive and negative connectives.

Definition 4 (Neutral/Positive/Negative connectives) In a formula $\varphi$, we will say that an occurrence of a connective is neutral if it is not a quantifier, positive (or synchronous) if it is a universal quantifier of polarity true or an existential quantifier of polarity false, negative (or asynchronous) if it is a universal quantifier of polarity true or an existential quantifier of polarity false.

Definition 5 (Monopoles/Bipoles) Monopoles are formula built from atoms with neutral and negative connectives. Bipoles are formula built from monopoles with neutral and positive connectives.

Bipoles, whose definition is adapted from [31] may be seen as a characterization of which formulæ can be used in $\mathcal{T h}_{2}$ in order to obtain a superdeduction system which is complete: If for some $\mathrm{R}: P \rightarrow \varphi \in \mathcal{T h}_{2}, \varphi$ is a bipole, then the rule $\mathrm{R}_{R}$ (focusing on $\varphi$ on the right) is complete. Dually if $\neg \varphi$ is a bipole, then the rule $\mathrm{R}_{L}$ (focusing on $\varphi$ on the left) is complete. These assertions are summarized in the following completeness theorem (extended from [11]).

Theorem 3 (Completeness of superdeduction modulo)

If for all $\varphi$ appearing in $P \rightarrow \varphi \in \mathcal{T h}_{2}, \varphi$ and $\neg \varphi$ are both bipoles, then $\mathcal{T} h_{1}, \mathcal{T} h_{2}, \Gamma \vdash \Delta$, implies $\Gamma \vdash{\stackrel{\equiv}{\perp_{1}}}^{+} \Delta$.

Proof. From the completeness of superdeduction which is proven in [11], for all $\varphi \in \mathcal{T} h_{2}$, there exists a proof of $\vdash^{+2} \varphi$. From the completeness of deduction modulo, for all $\varphi \in \mathcal{T} h_{1}$, there exists a proof of $\vdash_{\equiv_{1}} \varphi$. Starting from a proof of $\mathcal{T} h_{1}, \mathcal{T} h_{2}, \Gamma \vdash \Delta$, using cuts with the proofs of $\vdash_{\equiv_{1}^{+2}}^{+2}$ for all $\varphi \in \mathcal{T} h_{1} \cup \mathcal{T} h_{2}$, we get a proof of $\Gamma \stackrel{\vdash_{\equiv}^{+2}}{\rightleftarrows_{1}} \Delta$.

These pictures represent respectively a bipole, a formula whose negation is a bipole and a bipole whose negation is also a bipole. Minuses represent negative connectives, pluses represent positive connectives and the grey background represents neutral connectives.

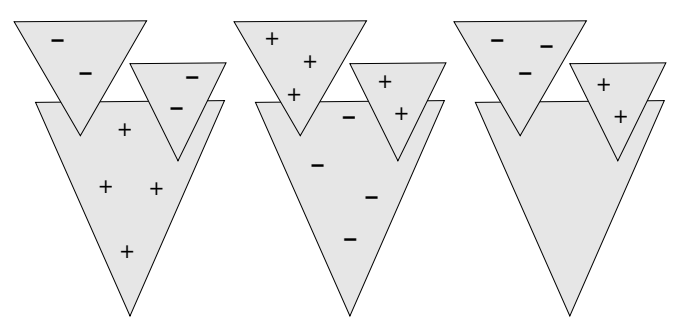


Finally a procedure, greatly inspired by Andreoli's Bipolarisation [31], has been proposed in [5] to transform any set of proposition rewrite rules into an equivalent one verifying this property, namely, for all $P \rightarrow \varphi \in \mathcal{T h}_{2}$, replacing $\varphi$ by its greatest prefix which is a bipole and whose negation is also a bipole; any subformula $\psi$ of $\varphi$ which is consequently separated from $\varphi$ is then replaced in the prefix by a fresh predicate symbol $Q$ parametrised by the free variables of $\psi$; finally the rule $Q \rightarrow \psi$ is recursively processed by the procedure and then added to $\mathcal{T h}_{2}$. For instance the proposition rewrite rule $P \rightarrow\left(\forall x A(x) \Rightarrow(\forall x . \exists y \cdot B(x, y))\right.$ is transformed into $\left\{\begin{array}{l}(\forall x \cdot A(x)) \Rightarrow(\forall x \cdot Q(x)) \\ Q(x) \rightarrow \exists y \cdot B(x, y)\end{array}\right.$. Consequently we make the following hypothesis for the rest of the paper. Hypothesis 1 If $P \rightarrow \varphi \in \mathcal{T} h_{2}$, then $\varphi$ and $\neg \varphi$ are both bipoles.

\section{Focalization in cut-free superdeduction modulo}

Using awkard theories to extend deduction through deduction modulo or superdeduction is known to jeopardize cut-elimination (completeness of the cut-free deduction system) and normalisation (termination of a cut-elimination procedure): For example if $A \equiv_{1,2} A \Rightarrow A$, one can easily build a proof of $\vdash_{\equiv_{1}^{+2}}^{+} A^{1}$, but there exists no cut-free proof of it. However cut-elimination and normalisation are well-studied for deduction modulo: Several criteria have been presented for theories which do not endanger cut-elimination or normalisation of the resulting deduction modulo system. Let us cite first the early work of Dowek and Werner [23], where the following theorem for classical sequent calculus modulo is proved.

Theorem 4 ([23]) If the light double negation of a rewrite system $\mathcal{R}$ has a premodel, cut-elimination holds for the classical sequent calculus modulo $\mathcal{R}$.

Both definitions of light double negation and pre-model can be found in [23]. Dowek also proposed in [24] an extension of Heyting algebras called truth values algebras which allows to distinguish provable equivalence from computational equivalence. A theory is said to be superconsistent if it has a model in all truth values algebras. Superconsistency of some theory has been proved in [24] and in $[25]$ to imply strong normalisation in the intuitionistic natural deduction modulo the theory. However this criterion does not apply here since we consider classical sequent calculus.

Hermant deeply studied cut-elimination for the intuitionistic and classical sequent calculus particularly using semantic methods in $[27,32,26]$. Among others, he proved the following cut-elimination theorem for classical sequent calculus modulo.

Theorem 5 ([26]) If $\mathcal{R}$ is a rewrite system compatible with a well-founded order, if $\mathcal{R}^{+}$is a positive rewrite system whose right-hand sides are $\mathcal{R}$-normal forms and if $\mathcal{R} \cup \mathcal{R}^{+}$is confluent, then cut-elimination holds for the classical sequent calculus modulo $\mathcal{R} \cup \mathcal{R}^{+}$.

\footnotetext{
${ }^{1}$ the proof may be written $(\lambda x . x x)(\lambda x . x x)$ in a $\lambda$-calculus style.
} 
The definition of a positive rewrite system can be found in [26]. Bonichon and Hermant also developed a constructive proof of cut-elimination for the intuitionistic sequent calculus modulo in [33].

Finally Burel and Kirchner proposed another approach in [28] where they use abstract canonical systems and abstract completion in order to mechanically transform a classical sequent calculus system into an equivalent one having the cut-elimination property.

Theorem 6 ([28]) A sequent has a proof in classical sequent calculus modulo some theory $\mathcal{R}$ if and only if it has a cut-free proof in the saturated theory corresponding to $\mathcal{R}$.

Our aim is now to relate superdeduction modulo to deduction modulo in order to prove that all these criteria are extendible to superdeduction modulo. We will only consider cut-free deduction systems and show that they allow to prove exactly the same sequents: A sequent $\Gamma \stackrel{c f+2}{\stackrel{\equiv}{=}_{1}} \Delta$ is provable if and only if $\Gamma \stackrel{c f}{{ }_{\equiv}}{ }_{1,2} \Delta$ is. The translation of a proof in superdeduction modulo into sheer deduction modulo is already shown in Lemma 1 . Now let us prove the converse: If $\Gamma \stackrel{c f}{\vdash_{\equiv}^{f}} \Delta$, then $\Gamma \stackrel{c f+_{\equiv_{1}}}{\equiv} \Delta$. To achieve this goal, we will first consider some proposition rewrite rule $\mathrm{R}: P \rightarrow \varphi$ of $\mathcal{T} h_{2}$ and prove that if $\Gamma \stackrel{c f++_{2}}{\equiv} \varphi, \Delta$,

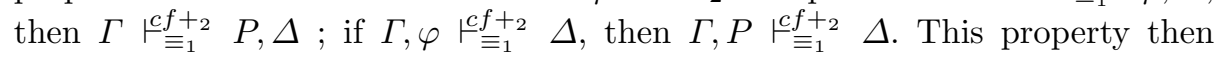
implies the admissibility of rules $\equiv_{2} \frac{\Gamma \vdash \varphi, \Delta}{\Gamma \vdash \psi, \Delta} \varphi \equiv_{2} \psi$ and $\equiv_{2} \frac{\Gamma, \varphi \vdash \Delta}{\Gamma, \psi \vdash \Delta} \varphi \equiv_{2} \psi$ in cut-free superdeduction modulo, which in turn implies that if $\Gamma \stackrel{c f}{\stackrel{N}{\equiv}_{1,2}} \Delta$, then $\Gamma \stackrel{c f++_{2}}{\stackrel{\equiv}{\equiv}_{1}} \Delta$. The proof of the property deals with permutability problems in classical sequent calculus: Indeed the intuition is to unite the steps of the proof that decompose $\varphi$. Considering the permutability problems that one can have when dealing with quantifiers (see Section 3), we can easily build a proof of some sequent $\Gamma \vdash_{\equiv_{1}}^{+2} \varphi, \Delta$ where the three steps decomposing $\varphi$, marked with the symbol $*$, cannot be united using sheer permutations:

$$
\begin{aligned}
& \forall_{L} \frac{\frac{\cdots}{A\left(y_{0}\right) \Rightarrow B\left(x_{0}\right), A\left(y_{0}\right) \vdash B\left(x_{0}\right)}}{A\left(y_{0}\right) \Rightarrow B\left(x_{0}\right),(\forall x \cdot A(x)) \vdash B\left(x_{0}\right)} * \\
& \forall_{L} \overline{\exists y \cdot\left(A(y) \Rightarrow B\left(x_{0}\right)\right),(\forall x . A(x)) \vdash B\left(x_{0}\right)} \\
& \forall_{L} \frac{\exists x \cdot \exists y \cdot(A(y) \Rightarrow B(x)),(\forall x \cdot A(x)) \vdash B\left(x_{0}\right)}{\forall} \\
& \Rightarrow_{R} \frac{\forall x \cdot \exists y \cdot(A(y) \Rightarrow B(x)),(\forall x \cdot A(x)) \vdash(\forall x \cdot B(x))}{\forall x . \exists y \cdot(A(y) \Rightarrow B(x)) \vdash(\forall x \cdot A(x)) \Rightarrow(\forall x \cdot B(x))} *
\end{aligned}
$$

where $\varphi$ is $(\forall x . A(x)) \Rightarrow(\forall x . B(x))$. Let us notice both $\varphi$ and $\neg \varphi$ are bipoles. A solution is to combine the permutations with contractions as follows. 


$$
\begin{aligned}
& \forall_{R} \frac{\frac{\cdots}{A\left(y_{0}\right) \Rightarrow B\left(x_{0}\right), A\left(x_{0}\right), A\left(y_{0}\right) \vdash B\left(x_{1}\right), B\left(x_{0}\right)}}{A\left(y_{0}\right) \Rightarrow B\left(x_{0}\right), A\left(x_{0}\right), A\left(y_{0}\right) \vdash \forall x \cdot B(x), B\left(x_{0}\right)}{ }_{L} \\
& \Rightarrow_{R} \frac{\frac{A\left(y_{0}\right) \Rightarrow B\left(x_{0}\right), A\left(x_{0}\right), \forall x \cdot A(x) \vdash \forall x \cdot B(x), B\left(x_{0}\right)}{\exists_{0}}}{\exists_{L} \frac{A\left(y_{0}\right) \Rightarrow B\left(x_{0}\right), A\left(x_{0}\right) \vdash \varphi, B\left(x_{0}\right)}{\forall}} * \\
& \forall_{L} \overline{\exists y \cdot A(y) \Rightarrow B\left(x_{0}\right), A\left(x_{0}\right) \vdash \varphi, B\left(x_{0}\right)} \\
& { }_{L} \frac{\forall x \cdot \exists y \cdot A(y) \Rightarrow B(x), A\left(x_{0}\right) \vdash \varphi, B\left(x_{0}\right)}{\forall x \cdot \exists y \cdot A(y) \Rightarrow B(x), \forall x \cdot A(x) \vdash \varphi, B\left(x_{0}\right)} * \\
& \forall_{R} \frac{\forall x \cdot \exists y \cdot A(y) \Rightarrow B(x), \forall x \cdot A(x) \vdash \varphi, B\left(x_{0}\right)}{\forall x \cdot \exists y \cdot A(x) \vdash \varphi, \forall x \cdot B(x)} * \\
& \begin{aligned}
\Rightarrow_{R} & \overline{\forall x \cdot \exists y \cdot A(y) \Rightarrow B(x) \vdash \varphi,(\forall x \cdot A(x)) \Rightarrow(\forall x \cdot B(x))} \\
\operatorname{CoNTR}_{R} & \frac{\forall x \cdot \exists y \cdot(A(y) \Rightarrow B(x)) \vdash(\forall x \cdot A(x)) \Rightarrow(\forall x \cdot B(x))}{\forall}
\end{aligned}
\end{aligned}
$$

In this proof, all the steps decomposing $(\forall x . A(x)) \Rightarrow(\forall x . B(x))$ have been duplicated and then united. It seems that this manipulation allows to transform any proof using several LK inference rules to decompose a formula $\varphi$ into a proof using several occurrences of a single superdeduction inference rule decomposing the corresponding predicate $P\left(P \rightarrow \varphi \in \mathcal{T} h_{2}\right)$. However proving that this manipulation always solves the problem remains an open question to our knowledge. We choose to avoid this kind of manipulation by making the following hypothesis.

Hypothesis 2 If $P \rightarrow \varphi \in \mathcal{T} h_{2}$, then $\varphi$ is in prenex normal form.

Let us notice that together with Hypothesis 1, it implies the following lemma.

Lemma 2 If $P \rightarrow \varphi \in \mathcal{T} h_{2}$, then $\varphi$ is either $\forall x_{1} \ldots x_{n} . \psi$ or $\exists x_{1} \ldots x_{n} . \psi$ for some propositional formula $\psi$. We will respectively say that $\varphi$ is right-handed and left-handed.

Hypotheses 2 and 1 can be replaced by the following single hypothesis (which we will call Hypothesis 3): For any $\varphi$ appearing in $P \rightarrow \varphi \in \mathcal{T} h_{2}$, either $\varphi$ or its negation is a monopole. Then the only quantifiers of $\varphi$ are either positive $\forall$ and negative $\exists$, or negative $\forall$ and positive $\exists$. Indeed in this case, one can always find a prenex normal form $\psi$ classically equivalent to $\varphi$ which satisfies Hypotheses 2 and 1 and such as the inference rules associated with $P \rightarrow \varphi$ are the same as the rules associated with $P \rightarrow \psi$. The deduction system is consequently unchanged and then our final result also holds if we just replace Hypotheses 2 and 1 by Hypothesis 3. Furthermore the procedure proposed in [5] which turns any set of proposition rewrite rules into an equivalent one verifying Hypothesis 1 can easily be strengthened into a procedure which turns any set of proposition rewrite rules into an equivalent one verifying this new single hypothesis.

Let us illustrate this with an example: The proposition rewrite rule $\mathrm{R}: P \rightarrow$ $(\forall x . A(x)) \Rightarrow(\exists y \cdot B(y))$ verifies Hypothesis 3 (which in turn implies Hypothesis 1$)$, but does not verify Hypothesis 2. However $(\forall x \cdot A(x)) \Rightarrow(\exists y \cdot B(y))$ is classically equivalent to $\exists x . \exists y .(A(x) \Rightarrow B(y))$. Furthermore we obtain the same superdeduction inference rules if we replace the proposition rewrite rule $\mathrm{R}$ by $\mathrm{R}^{\prime}: P \rightarrow \exists x . \exists y \cdot(A(x) \Rightarrow B(y))$, namely 


$$
\frac{\Gamma, A\left(t_{1}\right) \vdash B\left(t_{2}\right), \Delta}{\Gamma \vdash P, \Delta} \quad \frac{\Gamma, B(y) \vdash \Delta \quad \Gamma \vdash A(x), \Delta}{\Gamma, P \vdash \Delta} y \notin \mathcal{F} \mathcal{V}(\Gamma, \Delta)
$$

Finally $\mathrm{R}^{\prime}$ satisfies both Hypotheses 1 and 2 .

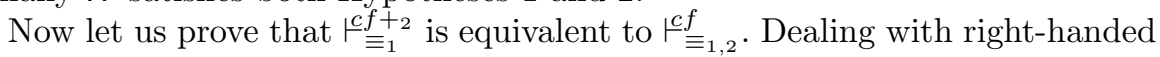
formulæ on the right and left-handed formulæ on the left of a sequent needs the following lemma, adapted from a central lemma in Hermant's semantic proofs of cut-elimination for deduction modulo [26].

Lemma 3 (Kleene's lemma adapted to $\vdash_{\equiv_{1}^{+2}}^{+2}$

Let $A_{1} \equiv_{1} A_{2} \equiv_{1} \ldots A_{n} \equiv_{1} \varphi$ be some propositions. Let $\Theta=A_{1}, A_{2} \ldots A_{n}$.

- If $\varphi=\neg A$ and $\Gamma, \Theta \stackrel{c f++_{\equiv_{1}}}{\equiv_{1}} \Delta$ then $\Gamma \stackrel{c f f++_{2}}{\equiv_{1}} A, \Delta$.

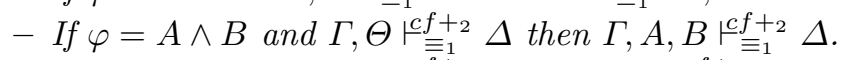

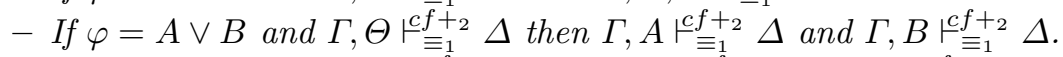

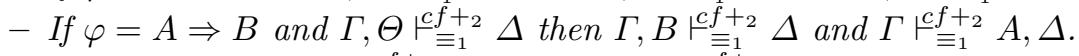

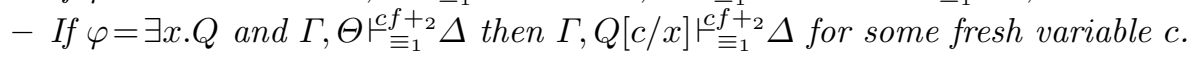

- If $\varphi=\neg A$ and $\Gamma \stackrel{c f++_{2}}{\equiv_{1}} \Theta, \Delta$ then $\Gamma, A \stackrel{c f+\digamma_{\equiv_{1}}}{=} \Delta$.

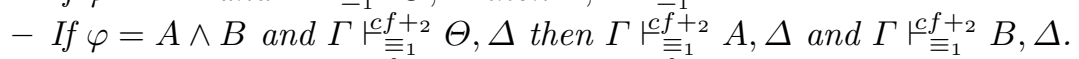

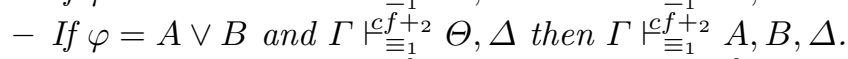

- If $\varphi=A \Rightarrow B$ and $\Gamma \stackrel{c f++_{2}}{\stackrel{c}{\equiv_{1}}} \Theta, \Delta$, then $\Gamma, A \stackrel{c f++_{2}}{\stackrel{\equiv}{1}_{1}} B, \Delta$.

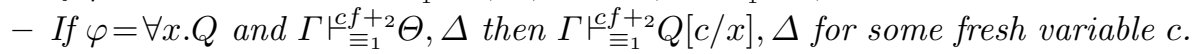

Proof. For each assertion, by induction on the derivation and detailed in Appendix A.

It implies the following lemma.

Lemma 4 Let us consider some $\mathrm{R}: P \rightarrow \varphi \in \mathcal{T} h_{2}$.

- If $\varphi$ is right-handed and $\Gamma \stackrel{c f++_{2}}{\equiv} \varphi, \Delta$, then there exists (cut-free) proofs of

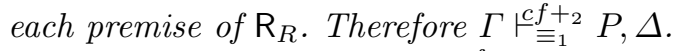

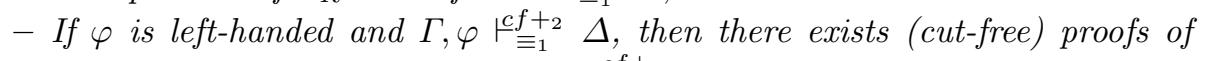

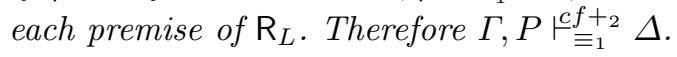

Proof. By iteration of Lemma 3. The proof is detailed in Appendix A. The idea is to unite the decomposition of $\varphi$ at the root of the proof: All these steps can move downward since $\varphi$ is right-handed and decomposed on the right. The proof is detailed in Appendix A.

Lemma 5 deals with right-handed formulæ on the left and left-handed formulæ on the right.

Lemma 5 Let us consider $\mathrm{R}: P \rightarrow \varphi \in \mathcal{T h}_{2}$.

- If $\varphi$ is right-handed and $\Gamma, \varphi \stackrel{c f++_{2}}{\equiv_{1}} \Delta$, then $\Gamma, P \stackrel{c f++_{2}}{\models_{1}} \Delta$.

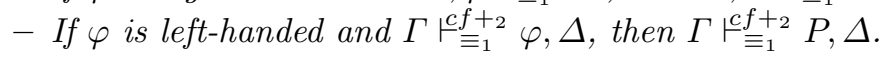

Proof. The proof is detailed in Appendix A. The idea is to fully decompose $\varphi$, partially eliminate contractions, and then to pull up the decomposition of $\varphi$. 
Lemmas 4 and 5 are concentrated in the following theorem, which proves that rewriting through $\mathcal{T} h_{1} \cup \mathcal{T} h_{2}$ preserves provability in superdeduction modulo.

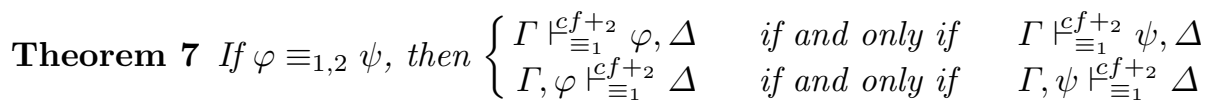

Proof. First by Lemmas 4 and 5, provability is preserved by one-step head reduction through $\mathcal{T h}_{2}$ (i.e. if $\varphi=P \sigma$ for some substitution $\sigma$ and $\psi=\phi \sigma$ and $\left.P \rightarrow \phi \in \mathcal{T} h_{2}\right)$. By induction on $\varphi$, we prove that it extends to any one-step reduction $\left(\varphi \rightarrow_{2} \psi\right)$. Then by induction on $\varphi \equiv_{1,2} \psi$, we obtain the final result.

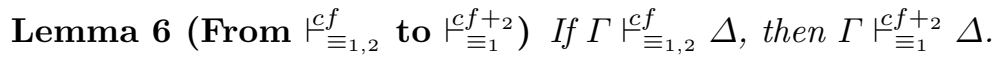

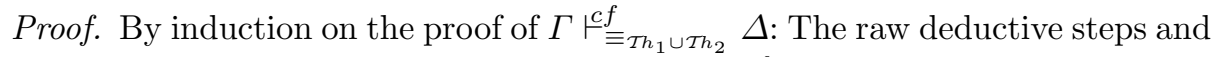

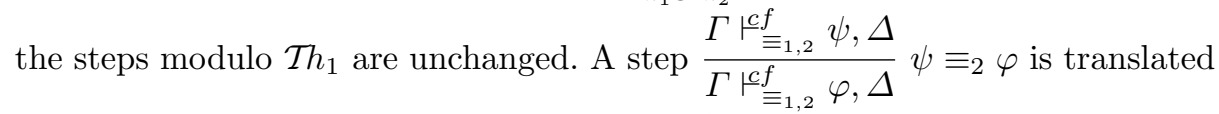
(by induction hypothesis) into a proof of $\Gamma \stackrel{c f+{ }^{\prime}+2}{\equiv_{1}} \psi, \Delta$ which is translated using

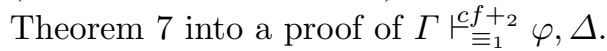

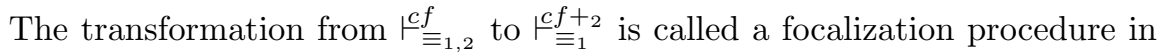
the focusing terminology. It transforms (cut-free) unstructured proofs into (cutfree) structured focusing proofs (superdeduction proofs here). In addition, using superdeduction rules instead of atomic deduction steps gives intuitive informations about the structure of proofs. We can therefore imagine building proofs only through superdeduction rules, as in the proof of the example of Section 2. An important difference with usual focalization is that in superdeduction, whenever this transformation has to focalize a sequence of connectives on one side of sequents, then it also has to focalize it on the other side of sequents, potentially in the same proof. Difficulties also arise from the fact that superdeduction modulo allows inferences to spawn new connectives from atoms. Finally we get the equivalence between cut-free deduction modulo and cut-free superdeduction modulo.

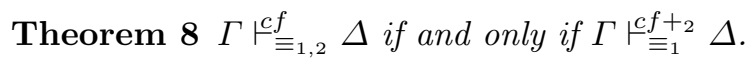

Proof. By Lemmas 1 and 6.

As a corollary we directly obtain that cut-elimination holds for deduction modulo $\mathcal{T} h_{1} \cup \mathcal{T} h_{2}$ if and only if it holds for the superdeduction modulo system associated with $\left(\mathcal{T} h_{1}, \mathcal{T} h_{2}\right)$. In particular it holds if $\left(\mathcal{T} h_{1}, \mathcal{T} h_{2}\right)$ verifies the criterion of Theorem 4,5 or 6 .

\section{A tableau method for superdeduction modulo}

An interesting use of the cut-elimination property for superdeduction modulo is to express the system through a sound and complete tableau method. In this section we choose to extend TaMeD - Tableau Method for Deduction modulo $[34,14]$ — whose rules are depicted in Figure 1. 
Tableau form transformation $\operatorname{Tab}(\mathcal{T})$

$$
\begin{aligned}
& \mathcal{B},\left(\varphi_{1} \vee \varphi_{2}\right)^{l}\left|\mathcal{T} \stackrel{t a b}{\longrightarrow} \mathcal{B}, \varphi_{1}^{l}\right| \mathcal{B}, \varphi_{2}^{l} \mid \mathcal{T} \\
& \mathcal{B}, \neg\left(\varphi_{1} \wedge \varphi_{2}\right)^{l}\left|\mathcal{T} \stackrel{t a b}{\longrightarrow} \mathcal{B}, \neg \varphi_{1}^{l}\right| \mathcal{B}, \neg \varphi_{2}^{l} \mid \mathcal{T} \\
& \mathcal{B},\left(\varphi_{1} \wedge \varphi_{2}\right)^{l}\left|\mathcal{T} \stackrel{t a b}{\longrightarrow} \mathcal{B}, \varphi_{1}^{l}, \varphi_{2}^{l}\right| \mathcal{T} \\
& \mathcal{B}, \neg\left(\varphi_{1} \vee \varphi_{2}\right)^{l}\left|\mathcal{T} \stackrel{t a b}{\longrightarrow} \mathcal{B}, \neg \varphi_{1}^{l}, \neg \varphi_{2}^{l}\right| \mathcal{T} \\
& \mathcal{B},\left(\varphi_{1} \Rightarrow \varphi_{2}\right)^{l}\left|\mathcal{T} \stackrel{t a b}{\longrightarrow} \mathcal{B}, \neg \varphi_{1}^{l}\right| \mathcal{B}, \varphi_{2}^{l} \mid \mathcal{T} \\
& \mathcal{B}, \neg\left(\varphi_{1} \Rightarrow \varphi_{2}\right)^{l}\left|\mathcal{T} \stackrel{t a b}{\longrightarrow} \mathcal{B}, \varphi_{1}^{l}, \neg \varphi_{2}^{l}\right| \mathcal{T} \\
& \mathcal{B},(\forall x . P)_{n_{x}}^{l}\left|\mathcal{T} \stackrel{t a b}{\longrightarrow} \mathcal{B},(\forall x . P)_{n_{x}-1}^{l}, P^{l, x}\right| \mathcal{T} \quad\left(x \text { fresh and } n_{x}>1\right) \\
& \mathcal{B}, \neg(\exists x . P)_{n_{x}}^{l}\left|\mathcal{T} \stackrel{t a b}{\longrightarrow} \mathcal{B}, \neg(\exists x . P)_{n_{x}-1}^{l}, \neg P^{l, x}\right| \mathcal{T} \quad\left(x \text { fresh and } n_{x}>1\right) \\
& \mathcal{B},(\exists x . P)^{l}\left|\mathcal{T} \stackrel{\text { tab }}{\longrightarrow} \mathcal{B},(P[f(l) / x])^{l}\right| \mathcal{T} \quad \text { ( } f \text { fresh skolem symbol) } \\
& \mathcal{B}, \neg(\forall x . P)^{l}\left|\mathcal{T} \stackrel{\text { tab }}{\longrightarrow} \mathcal{B},(\neg P[f(l) / x])^{l}\right| \mathcal{T} \quad(f \text { fresh skolem symbol }) \\
& \text { TaMeD rules } \frac{\mathcal{B}, \varphi_{1}, \neg \varphi_{2} \mid \mathcal{T}[C]}{\mathcal{T}\left[C \cup\left\{\varphi_{1} \stackrel{?}{=} \varphi_{2}\right\}\right]} \quad \frac{\mathcal{B}, U \mid \mathcal{T}[C]}{\mathcal{T}^{\prime} \mid \mathcal{T} \quad\left[C \cup\left\{U_{\mid w} \stackrel{?}{=} l\right\}\right]} \\
& \text { if } l \rightarrow_{1} r, U_{\mid w} \text { is an atomic proposition and } \mathcal{T}^{\prime}=\operatorname{Tab}\left(\mathcal{B}, U[r]_{w}\right) \\
& \begin{aligned}
& \mathcal{B}, \neg \perp^{l} \mid \mathcal{T} \stackrel{t a b}{\longrightarrow} \mathcal{B} \mid \mathcal{T} \\
& \mathcal{B}, \top^{l} \mid \mathcal{T} \stackrel{t a b}{\longrightarrow} \mathcal{B} \mid \mathcal{T} \\
& \mathcal{B}, \neg \neg \varphi^{l} \mid \mathcal{T} \stackrel{t a b}{\longrightarrow} \mathcal{B}, \varphi^{l} \mid \mathcal{T} \\
& \mathcal{B}, \perp^{l} \mid \mathcal{T} \stackrel{t a b}{\longrightarrow} \mathcal{T} \\
& \mathcal{B}, \neg \top^{l} \mid \mathcal{T} \stackrel{t a b}{\longrightarrow} \mathcal{T}
\end{aligned}
\end{aligned}
$$

Fig. 1. Tableaux for Deduction Modulo.

We use the presentation of TaMeD of [34], where each formula $\varphi$ is annotated with a set of first-order variables $l$, denoted $\varphi^{l}$. This set at least contains the free variables of $\varphi$. In addition, each formula $\psi$ of the form $\forall x . \varphi$ or $\neg(\exists x . \varphi)$ is annotated with an integer $n$, denoted $\psi_{n}$. This integer is the maximal number of decomposition of $\psi$ that are allowed, in order to keep the method terminating.

The extension of TaMeD is done in the following manner. Each superdeduction rule on the left

is translated into $\quad \mathcal{B}_{1}, P_{n_{P}}^{l} \mid \mathcal{T} \quad \stackrel{t a b}{\longrightarrow}$

$$
\frac{\left(\Gamma, \Gamma_{i} \vdash \Delta_{i}, \Delta\right)_{1 \leqslant i \leqslant n}}{\Gamma, P \vdash \Delta} X \cap \mathcal{F} \mathcal{V}(\Gamma, \Delta)=\emptyset
$$

$$
\mathcal{B}_{1}, P_{n_{P}-1}^{l}, \Gamma_{1}^{\prime l \cup Y} \sigma, \neg \Delta_{1}^{\prime l \cup Y} \sigma|\cdots| \mathcal{B}_{1}, P_{n_{P}-1}^{l}, \Gamma_{n}^{\prime l \cup Y} \sigma, \neg \Delta_{n}^{\prime l \cup Y} \sigma \mid \mathcal{T} \quad \text { if } n_{P}>0
$$

where $\Gamma_{i}^{\prime}$ and $\Delta_{i}^{\prime}$ stand respectively for $\Gamma_{i}$ and $\Delta_{i}$ where each meta-term (such as $t$ in the $\exists_{R}$-rule) is replaced by a fresh variable, $Y$ stands for the set of these fresh variables, and $\sigma$ stands for a substitution replacing each variable $x_{i}$ of $X=x_{1}, x_{2}, \ldots$ by a fresh skolem function $f_{i}(l)$. The construction of the tableau rule corresponding to superdeduction rules on the right is done in a similar way.

Together with the rules of Figure 1, we obtain a sound and complete tableau method for superdeduction modulo, provided that the superdeduction modulo system enjoys cut-elimination: Each use of a superdeduction tableau transformation can be translated into a use of the corresponding superdeduction inference rule and conversely. Since it also holds for the other rules of TaMeD (soundness and completeness of $\mathrm{TaMeD}[34,14])$, we consequently obtain the equivalence between the superdeduction modulo tableau method and the corresponding cutfree superdeduction modulo system. 
Let us show how it operates on the example of Section 2 The tableau rule which is associated with the $\mathbb{N}_{L}$ inference rule is

$$
\begin{aligned}
\mathcal{B}, \mathbb{N}(n)_{n}^{l} \mid \mathcal{T} \stackrel{t a b}{\longrightarrow} & \mathcal{B}, \mathbb{N}(n)_{n-1}^{l}, \neg(0 \in Y)^{l \cup\{Y\}} \mid \mathcal{B}, \mathbb{N}(n)_{n-1}^{l}, \neg(\mathfrak{H}(Y))^{l \cup\{Y\}} \\
& \left|\mathcal{B}, \mathbb{N}(n)_{n-1}^{l},(n \in Y)^{l \cup\{Y\}}\right| \mathcal{T}
\end{aligned}
$$

It corresponds to the first step of the proof in the example of Section 2, except that a first-order variable $Y$ replaces the first-order constant $\tilde{P}$ which relates to $\operatorname{sum}(x)=x * x$. However $Y$ may be instantiated to $\tilde{P}$ through the extended narrowing rule (see [34] or Figure 1)

$$
\frac{\mathcal{B}, U \mid \mathcal{T} \quad[C]}{\mathcal{T}^{\prime} \mid \mathcal{T} \quad\left[C \cup\left\{U_{\mid w} \stackrel{?}{=} l\right\}\right]}\left\{\begin{array}{l}
l \rightarrow_{1} r \\
U_{\mid w} \text { is an atomic proposition } \\
\mathcal{T}^{\prime}=\operatorname{Tab}\left(\mathcal{B}, U[r]_{w}\right)
\end{array}\right.
$$

where $U_{\mid w}$ denotes the subformula of $U$ at some position $w$ and $U[r]_{w}$ denotes $U$ where this subformula at this position is replaced by $r$. We can use this TaMeD rule to instantiate $Y$ to $\tilde{P}$ in any $t \in Y$ which may appear, using the fact that $t \in \tilde{P} \rightarrow_{1} P(t)$. The generated constraint $\left(U_{\mid w} \stackrel{?}{=} l\right)$ is then $t \in Y \stackrel{?}{=} t \in \tilde{P}$ which will always be equivalent to $Y \stackrel{?}{=} \tilde{P}$.

\section{Conclusion}

Superdeduction modulo is the combination of superdeduction and deduction modulo with both inference rules systematically derived from an axiomatic theory and the ability to conduct deduction modulo computation. In this paper, we have filled the gap with the focusing approach by proposing an extension of classical sequent calculus and by showing that both focusing and superdeduction are instances of this extension. Our analysis then indicates that superdeduction stands in fact for introducing focusing phases which are directed by the unfolding of axioms, and which are made explicit in the explicit superdeduction system we have presented. Then we proved a focalization result for superdeduction, namely that cut-free deduction modulo can be translated into cut-free superdeduction modulo using permutations of the applications of the inference rules, provided that some hypothesis on the synchrony of the superdeduction axioms are verified. The inverse translation being trivial, we acquired as a corollary that cut-elimination for superdeduction modulo is equivalent to cut-elimination for deduction modulo, consequently obtaining that the numerous criteria for cutelimination in deduction modulo also hold for superdeduction modulo. Finally we proposed a tableau method for superdeduction modulo which is sound and complete provided that the corresponding deduction system enjoys cut-elimination.

Our comparison of superdeduction and focusing is meant to be carried forward. In particular we believe that our focalization proof is rather complicated. This is greatly due to the fact that our proof handles superdeduction modulo, consequently allowing inference rules to spawn new connectives from atoms. However we wish to compare our focalization proof with Andreoli's original one and with the elegant focalization graph technique introduced by Miller and Saurin in [35]. 
This analysis could lead us to simpler focalization proofs and weaker conditions for deductive theories which can be safely used in superdeduction modulo.

Superdeduction modulo is a promising framework for proof engineering. Our tableau method may be in particular the foundation of automated proof construction procedures based on superdeduction modulo. Besides let us notice that superdeduction modulo is already the core of a small proof assistant named Lemuridæ which can be downloaded at http://rho.loria.fr/lemuridae.html . Superdeduction modulo is also used in [22] in a restricted manner since modulo is only used on first-order terms (and not first-order propositions). Its expressiveness is nevertheless demonstrated by an encoding of functional PTS.

Building bridges between superdeduction and deduction modulo is also done in a related approach by Brauner and Dowek [36]. Whereas we proved the equivalence of cut-elimination for superdeduction modulo and deduction modulo, they proved the equivalence of normalisation for deduction modulo and supernatural deduction. Superdeduction and supernatural deduction stand for the same paradigm applied to classical sequent calculus for the first and to intuitionistic natural deduction for the second. However in this latter system, permutability problems forbid the paradigm to handle disjunctions or existential quantifications. Therefore an interesting extension would be to apply their approach to (classical sequent calculus) superdeduction.

Acknowledgements: The author thanks Claude Kirchner for his useful comments and advices. Many thanks also to Richard Bonichon and Cody Roux for fertile

discussions, to anonymous referees for their comments on a previous version of this paper, to the Modulo meetings and to the Pareo team for many interactions.

\section{References}

1. Huang, X.: Reconstruction proofs at the assertion level. In: Proceedings of CADE. Volume 814 of LNCS., Springer (1994) 738-752

2. Negri, S., von Plato, J.: Cut elimination in the presence of axioms. Bulletin of Symbolic Logic 4(4) (1998) 418-435

3. Hallnäs, L., Schroeder-Heister, P.: A proof-theoretic approach to logic programming. II. programs as definitions. Journal of Logic and Computation 1(5) (1991) $635-660$

4. McDowell, R., Miller, D.: Cut-elimination for a logic with definitions and induction. Theoretical Computer Science 232(1-2) (2000) 91-119

5. Brauner, P., Houtmann, C., Kirchner, C.: Principles of superdeduction. In Ong, L., ed.: Proceedings of LICS. (jul 2007) 41-50

6. Andreoli, J.M.: Logic programming with focusing proofs in linear logic. Journal of Logic and Computation 2(3) (1992) 297-347

7. Wack, B.: Typage et déduction dans le calcul de réécriture. PhD thesis, Université Henri Poincaré, Nancy 1 (October 2005)

8. Cirstea, H., Kirchner, C.: The rewriting calculus - Part I and II. Logic Journal of the Interest Group in Pure and Applied Logics 9(3) (May 2001) 427-498

9. Cirstea, H., Liquori, L., Wack, B.: Rewriting calculus with fixpoints: Untyped and first-order systems. In: TYPES. (2003) 147-161 
10. Urban, C.: Classical Logic and Computation. PhD thesis, University of Cambridge (October 2000)

11. Brauner, P., Houtmann, C., Kirchner, C.: Superdeduction at work. In: Rewriting, Computation and Proof. (2007) 132-166

12. Dowek, G., Hardin, T., Kirchner, C.: Theorem proving modulo. Journal of Automated Reasoning 31(1) (Nov 2003) 33-72

13. Dowek, G.: Confluence as a cut elimination property. In: RTA. (2003) 2-13

14. Bonichon, R., Hermant, O.: A semantic completeness proof for TaMeD. In: LPAR. (2006) 167-181

15. Dowek, G., Werner, B.: Arithmetic as a theory modulo. In: RTA. (2005) 423-437

16. Allali, L.: Algorithmic equality in Heyting arithmetic modulo. In: TYPES. (2007) To appear.

17. Dowek, G., Miquel, A.: Cut elimination for Zermelo's set theory. Available on author's web page

18. Dowek, G., Hardin, T., Kirchner, C.: HOL- $\lambda \sigma$ : an intentional first-order expression of higher-order logic. Math. Struct. in Comp. Science 11(1) (2001) 21-45

19. Dowek, G.: Proof normalization for a first-order formulation of higher-order logic. In: TPHOLs. (1997) 105-119

20. Cousineau, D., Dowek, G.: Embedding pure type systems in the lambda-pi-calculus modulo. In: TLCA. (2007) 102-117

21. Burel, G.: Unbounded proof-length speed-up in deduction modulo. In: CSL. (2007)

22. Burel, G.: Superdeduction as a logical framework. Accepted at LICS'08 (2008)

23. Dowek, G., Werner, B.: Proof normalization modulo. Journal of Symbolic Logic 68(4) (2003) 1289-1316

24. Dowek, G.: Truth values algebras and proof normalization. In: TYPES. (2006)

25. Dowek, G., Hermant, O.: A simple proof that super-consistency implies cut elimination. In: RTA. (2007) 93-106

26. Hermant, O.: Méthodes Sémantiques en Déduction Modulo. PhD thesis, École Polytechnique (2005)

27. Hermant, O.: Semantic cut elimination in the intuitionistic sequent calculus. In: TLCA. (2005) 221-233

28. Burel, G., Kirchner, C.: Cut elimination in deduction modulo by abstract completion. In: LFCS. (2007) 115-131

29. Dowek, G.: La part du calcul. Mémoire d'habilitation, Université de Paris 7 (1999)

30. Liang, C., Miller, D.: Focusing and polarization in linear, intuitionistic, and classical logic. Accepted (March 2008) to TCS (2008)

31. Andreoli, J.M.: Focussing and proof construction. Annals Pure Applied Logic 107(1-3) (2001) 131-163

32. Hermant, O.: A model-based cut elimination proof. In: 2nd St-Petersburg Days of Logic and Computability. (2003)

33. Bonichon, R., Hermant, O.: On constructive cut admissibility in deduction modulo. In: TYPES. (2006) 33-47

34. Bonichon, R.: TaMeD: A tableau method for deduction modulo. In: IJCAR. (2004)

35. Miller, D., Saurin, A.: From proofs to focused proofs: A modular proof of focalization in linear logic. In: CSL. (2007) 405-419

36. Brauner, P., Dowek, G., Wack, B.: Normalization in supernatural deduction and in deduction modulo. Available on the author's webpage (2007) 


\section{A Proofs of theorems and lemmas}

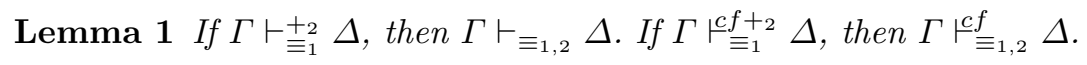

Proof. For some R : $P \rightarrow_{2} \varphi \in \mathcal{T} h_{2}$ the superdeduction step $\mathrm{R}_{R} \frac{H_{1} H_{2} \ldots H_{n}}{\Gamma \vdash_{\equiv}^{+2} P, \Delta}$ can be translated by definition into

$$
\frac{H_{1} H_{2} \ldots H_{n}}{\frac{[\text { Calc }]}{\Gamma \vdash_{\equiv_{1,2}} \varphi, \Delta}} \frac{\vdash_{\equiv_{1,2}} P, \Delta}{P \equiv_{2} \varphi}
$$

and similarly for superdeduction steps on the left.

In the rest of this appendix, we use the second presentation of deduction modulo (i.e. not the $\equiv_{1 R} / \equiv_{1 L}$ one).

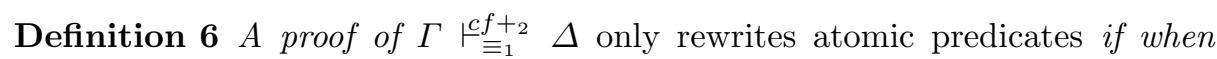
using rules such as

$$
\wedge_{R} \frac{\Gamma \vdash \psi_{1}, \Delta \quad \Gamma \vdash \psi_{2}, \Delta}{\Gamma \vdash \varphi, \Delta} \varphi \equiv_{1} \psi_{1} \wedge \psi_{2} \quad \wedge_{L} \frac{\Gamma, \psi_{1}, \psi_{2} \vdash \Delta}{\Gamma, \varphi \vdash \Delta} \varphi \equiv_{1} \psi_{1} \wedge \psi_{2}
$$

$\varphi$ is either an atomic predicate or syntactically equal to $\psi_{1} \wedge \psi_{2}$, and similarly for all the rules of the cut-free deduction system, except axioms.

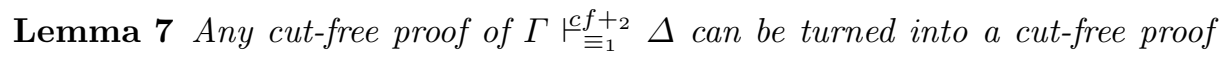
of the same sequent which only rewrites atomic predicates.

Proof. By induction on the proof:

axiom the proof already only rewrites atomic predicates.

conjunction on the right the proof is

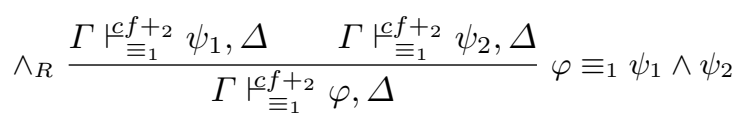

Since $\varphi \equiv_{1} \psi_{1} \wedge \psi_{2}$, since $\mathcal{T} h_{1}$ is confluent and since $\mathcal{T} h_{1}$ only rewrites atomic predicates, either $\varphi=\varphi_{1} \wedge \varphi_{2}$ with $\varphi_{i} \equiv_{1} \psi_{i}$, either $\varphi$ is atomic.

- If $\varphi$ is atomic, then by induction hypothesis, we obtain proofs of $\Gamma \stackrel{c f++_{2}}{\stackrel{\equiv}{1}_{1}}$

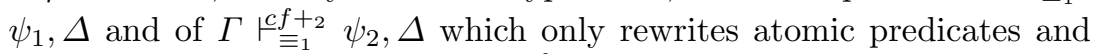
through $\wedge_{R}$ we get a proof of $\Gamma \stackrel{c f+{ }_{\equiv_{1}}}{\stackrel{1}{1}_{1}} \varphi, \Delta$ which only rewrites atomic predicates.

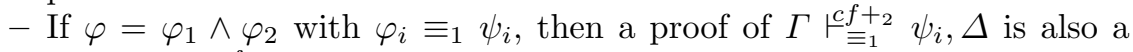

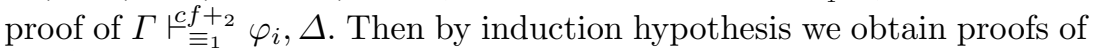
$\Gamma \stackrel{c f++_{2}}{\stackrel{\equiv}{\equiv}_{1}} \varphi_{i}, \Delta$ which only rewrite atomic predicates and we get through $\wedge_{R}$ a proof of $\Gamma \stackrel{c f f+2}{\stackrel{\equiv}{\equiv}_{1}} \varphi, \Delta$ which only rewrites atomic predicates. 
other cases are handled the same way.

Definition $7 \Gamma^{\prime}, \Delta^{\prime}$ is fully decomposed in some proof of $\Gamma, \Gamma^{\prime} \stackrel{c f++_{2}+_{1}}{{ }_{1}} \Delta^{\prime}, \Delta$ if this proof only rewrites atomic predicates and when applying Ax-rules in this proof, no logical connective of $\Gamma^{\prime}, \Delta^{\prime}$ remain (they have been fully decomposed).

Let us notice that if a proof only rewrites atomic predicates, the only way a logical connective of $\Gamma^{\prime}, \Delta^{\prime}$ can disappear is if it is decomposed by some rule of LK. Let us first prove the following auxiliary lemma.

Lemma 8 A proof of $\Gamma \stackrel{c f f+_{2}}{\models_{1}} \Delta$ can be transformed into a proof of the same sequent where $\Gamma, \Delta$ is fully decomposed.

Proof. First by Lemma 7 , we can suppose that the proof of $\Gamma \stackrel{c f+2}{\equiv_{1}} \Delta$ only rewrites atomic predicates. Then we proceed by induction on the multiset containing the number of logical connectives of $\Gamma, \Delta$ that remains in an Ax-rule, for each Ax-rule of the proof.

- If no logical connective of $\Gamma, \Delta$ remain, the proof is fully decomposed.

- If a logical connective of $\Gamma, \Delta$ appears in a principal formula of an Ax-rule, for instance a conjunction on the right:

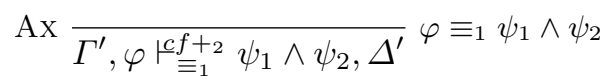

Since $\mathcal{T} h_{1}$ only rewrites atomic predicates and is confluent, either $\varphi$ is atomic, either $\varphi=\varphi_{1} \wedge \varphi_{2}$ with $\varphi_{i} \equiv_{1} \psi_{i}$.

In the first case the corresponding subproof can be turned into

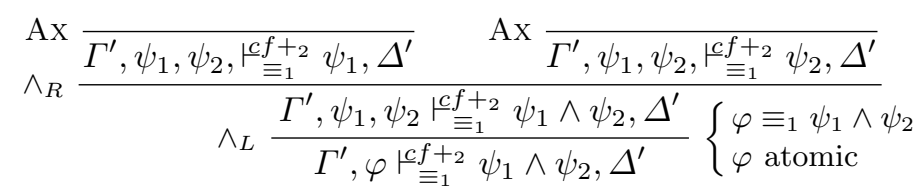

Since $\varphi$ is atomic, the modified proof still only rewrites atomic predicates.

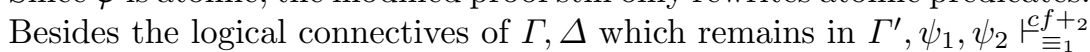
$\psi_{i}, \Delta^{\prime}$ for some $i \in\{1,2\}$ are those of $\Gamma^{\prime}, \Delta^{\prime}$ and $\psi_{i}$. (The occurrence of $\psi_{1}, \psi_{2}$ on the left-hand side must not be taken into account since it comes from the decomposition of $\varphi$ which is atomic.) These logical connectives consequently already appeared in the Ax-rule which we are replacing, and which also contains an additionnal logical connective of $\Gamma, \Delta$ (the $\wedge$ we are decomposing). Therefore the induction hypothesis may be applied on the global proof of $\Gamma \stackrel{c f+{ }_{1}}{=} \Delta$ obtaining consequently a proof of $\Gamma \stackrel{c f+_{2}}{\equiv{ }_{1}} \Delta$ which fully decomposes $\Gamma, \Delta$.

In the second case $\left(\varphi=\varphi_{1} \wedge \varphi_{2}\right)$, the corresponding subproof can be turned into

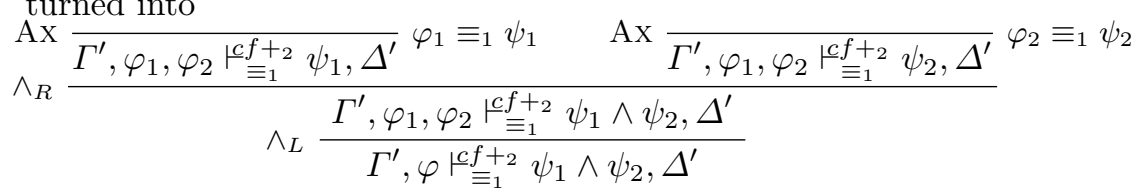


The modified proof still only rewrites atomic predicates. Besides there are fewer logical connectives of $\Gamma, \Delta$ in a sequent $\Gamma^{\prime}, \varphi_{1}, \varphi_{2} \stackrel{c f+_{2}}{\equiv_{1}} \psi_{i}$ for some $i \in\{1,2\}$ than in $\Gamma^{\prime}, \varphi \stackrel{c f++_{2}}{\equiv_{1}} \psi_{1} \wedge \psi_{2}, \Delta^{\prime}$. Therefore the induction hypothesis may be applied on the global proof of $\Gamma \stackrel{c f++_{2}}{\equiv} \Delta$ obtaining consequently a proof of $\Gamma \stackrel{c f+2}{\equiv_{1}} \Delta$ which fully decomposes $\Gamma, \Delta$.

- If a logical connective appears in a non-principal formula of an Ax-rule, for instance a disjunction on the right:

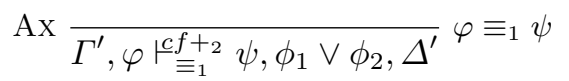

Then the corresponding subproof can be turned into

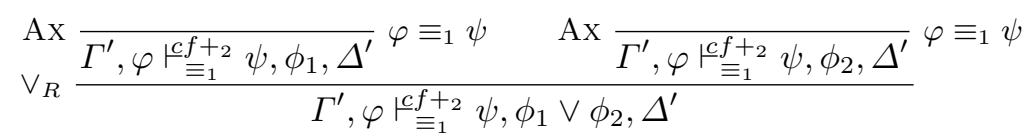

The modified proof still only rewrites atomic predicates. Besides there are

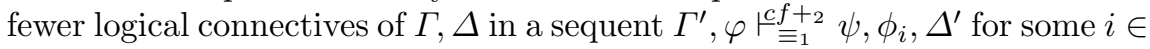
$\{1,2\}$ than in $\Gamma^{\prime}, \varphi \stackrel{c f+_{2}}{\equiv_{1}} \psi, \phi_{1} \vee \phi_{2}, \Delta^{\prime}$. Therefore the induction hypothesis may be applied on the global proof of $\Gamma \stackrel{c f+_{2}}{\equiv_{1}} \Delta$ obtaining consequently a proof of $\Gamma \stackrel{c f++_{2}}{{ }_{1}} \Delta$ which fully decomposes $\Gamma, \Delta$.

Definition 8 - A partial decomposition of a right-handed formula $\forall x_{1} \ldots x_{n} \cdot \varphi$ is a formula $\forall x_{k} \ldots x_{n} . \varphi \sigma$ for some substitution $\sigma$ on the variables $x_{1} \ldots x_{k-1}$. - A partial decomposition of a left-handed formula $\exists x_{1} \ldots x_{n} \cdot \varphi$ is a formula $\exists x_{k} \ldots x_{n} . \varphi \sigma$ for some substitution $\sigma$ on the variables $x_{1} \ldots x_{k-1}$.

Definition 9 A right-handed formula $\varphi=\forall x_{1} \ldots x_{n} . \phi$ is non-contracted in a proof of $\Gamma, \varphi \ldots \varphi \stackrel{c \stackrel{c f+2}{\equiv}{ }_{1}}{=} \Delta$ if no contraction is done on some formula $\forall x_{k} \ldots x_{n} . \phi \sigma$ coming from the partial decomposition of $\varphi$. A left-handed formula $\varphi=\exists x_{1} \ldots x_{n}$. $\phi$ is non-contracted in a proof of $\Gamma \stackrel{c f+2}{\equiv_{1}} \varphi \ldots \varphi, \Delta$ if no contraction is done on some formula $\exists x_{k} \ldots x_{n} . \phi \sigma$ coming from the partial decomposition of $\varphi$.

Lemma 9 Any proof of $\Gamma, \varphi_{1} \ldots \varphi_{n} \stackrel{c f+_{2}}{\equiv} \Delta$ where $\varphi_{i}$ is right-handed and fully decomposed can be transformed into a proof of $\Gamma, \varphi_{1} \ldots \varphi_{1}, \varphi_{2} \ldots \varphi_{2} \ldots \varphi_{n} \stackrel{c f++_{2}}{\equiv_{1}}$ $\Delta$ where each $\varphi_{i}$ is fully decomposed and non-contracted.

The same holds for left-handed formula on the right.

Proof. The proof is conducted by induction on the proof of $\Gamma, \varphi_{1} \ldots \varphi_{n} \stackrel{c f+_{2}}{\equiv} \equiv_{1}$. axiom on $\varphi_{i}$ :

$$
\mathrm{Ax} \frac{}{\Gamma, \varphi_{1} \ldots \varphi_{n} \stackrel{c f+2}{\sum_{1}} \psi, \Delta^{\prime}} \varphi_{i} \equiv_{1} \psi
$$

The $\varphi_{k}$ are already non-contracted in the proof. axiom on $\Gamma, \Delta$ :

$$
\operatorname{Ax} \frac{}{\Gamma^{\prime}, \psi_{1}, \varphi_{1} \ldots \varphi_{n} \stackrel{c f+2}{\equiv_{1}} \psi_{2}, \Delta} \psi_{1} \equiv_{1} \psi_{2}
$$

The $\varphi_{k}$ is already non-contracted in the proof. 
contraction on some formula of $\Gamma$ or $\Delta$ : We just apply the induction hypothesis.

contraction on some $\varphi_{i}(i=1$ without loss of generality):

$$
\frac{\Gamma, \varphi_{1}, \varphi_{1} \ldots \varphi_{n}, \varphi \stackrel{c f++_{2}}{\equiv} \Delta}{\Gamma, \varphi_{1} \ldots \varphi_{n} \stackrel{c f+f_{1}}{\equiv_{1}} \Delta}
$$

By induction hypothesis applied on the proof of $\Gamma, \varphi_{1}, \varphi_{1} \ldots \varphi_{n} \stackrel{c f++_{2}}{=} \Delta$, we obtain the proof where each $\varphi_{i}$ is fully decomposed and non-contracted.

decomposition of some formula of $\Gamma, \Delta$ : we just apply the induction hypothesis on the premises.

decomposition of some $\varphi_{i}=\forall x_{1} \ldots x_{n} . \phi$ ( $i=1$ without loss of generality):

$$
\frac{\Gamma, \forall x_{2} \ldots x_{n} . \phi\left[t / x_{1}\right], \varphi_{2} \ldots \varphi_{n} \stackrel{c f++_{2}}{\equiv{ }_{1}} \Delta}{\Gamma, \varphi_{1}, \varphi_{2} \ldots \varphi_{n} \stackrel{f f++_{2}}{\equiv_{1}} \Delta} \forall_{L}
$$

Then by induction hypothesis, we obtain a proof of

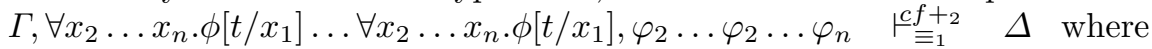
$\forall x_{2} \ldots x_{n} . \phi[t / x]$ and each $\varphi_{i}(i>1)$ is fully decomposed and non-contracted.

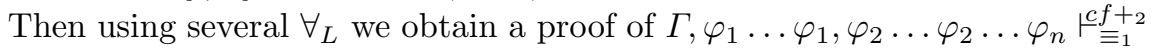
$\Delta$.

decomposition of some propositional $\varphi_{i}$ ( $i=1$ without loss of generality): it is sufficient to apply the induction on each premiss, obtaining proofs where each $\varphi_{i}(i>1)$ is non-contracted and fully decomposed. Then using the same inference rule we obtain a proof of $\Gamma, \varphi_{1} \ldots \varphi_{1}, \varphi_{2} \ldots \varphi_{2} \ldots \varphi_{n} \stackrel{c f+2}{\equiv_{1}} \Delta$ where each $\varphi_{i}$ is non-contracted and fully decomposed.

Let us prove Lemma 3.

\section{Lemma 3}

Let $A_{1} \equiv_{1} A_{2} \equiv_{1} \ldots A_{n} \equiv_{1} \varphi$ be some propositions. Let $\Theta=A_{1}, A_{2} \ldots A_{n}$.

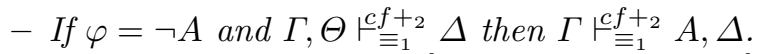

- If $\varphi=A \wedge B$ and $\Gamma, \Theta \stackrel{c f+\digamma_{\equiv}+2}{\equiv} \Delta$ then $\Gamma, A, B \stackrel{c f++_{1}}{\equiv_{1}} \Delta$.

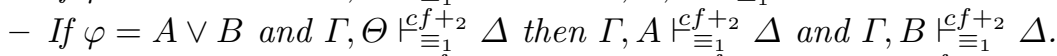

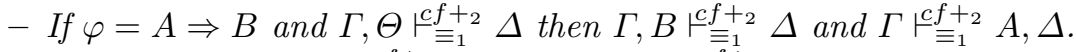

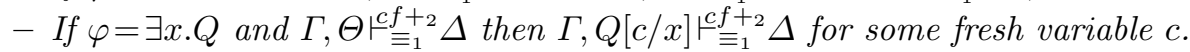

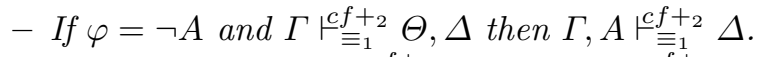

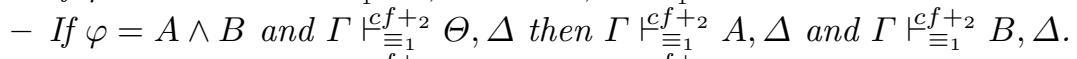

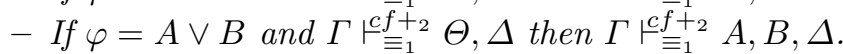

- If $\varphi=A \Rightarrow B$ and $\Gamma \stackrel{c f++_{2}}{\equiv_{1}} \Theta, \Delta$, then $\Gamma, A \stackrel{c f++_{\equiv_{1}}}{\equiv} B, \Delta$.

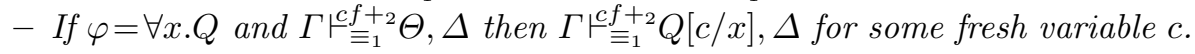

Proof. Let us prove the first proposition by induction on the proof of $\Gamma, \Theta \stackrel{c f+{ }_{1}+2}{\equiv} \Delta$. 
If the last step of the proof of $\Gamma, \Theta \stackrel{c f \perp_{1}}{\equiv_{1}} \Delta$ decomposes $A_{i} \equiv_{1} \neg A$ (we suppose without loss of generality that $i=1$ ), then since $\mathcal{T} h_{1}$ is confluent and only rewrites atomic propositions and first-order terms, the decomposition of $A_{1}$ is consequently $\neg L$, and we have a proof of $\Gamma, A_{2} \ldots A_{n} \stackrel{c f+_{2}}{=} A_{1}^{\prime}, \Delta$ with $A \equiv_{1} A^{\prime}$. Therefore by induction hypothesis, we obtain a proof of $\Gamma \stackrel{c f++_{2}}{\equiv_{1}} A, A^{\prime}, \Delta$ and using a contraction, we finally get a proof of $\Gamma \stackrel{c f \stackrel{1}{F}_{1}}{=} A, \Delta$ (since $A^{\prime} \equiv_{1} A$ ).

Otherwise let us consider the inference rule applied at the root of the proof.

contraction the contraction may be applied on a formula of $\Theta, \Gamma$ or $\Delta$, but in all cases it is sufficient to apply the induction hypothesis on the premiss of the contraction.

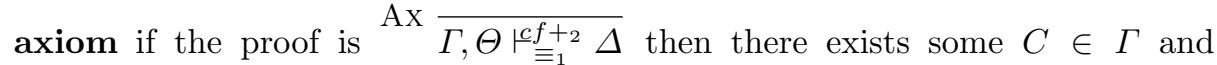
$D \in \Delta$ such that $C \equiv_{1} D$ or some $E \equiv_{1} A_{i}$ appears in $\Delta$. In the first

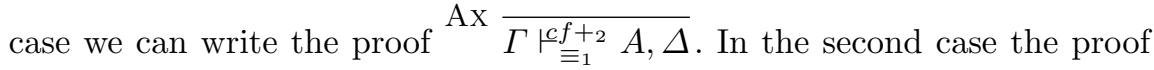
Ax $\overline{\Gamma, \Theta \vdash_{\equiv_{1}}^{c f+2} E, \Delta^{\prime}}$ where $\Delta=E, \Delta^{\prime}$ can be transformed into

$$
\operatorname{Ax} \frac{\overline{\Gamma, A \vdash_{\equiv_{1}}^{c f+2} A, \Delta^{\prime}}}{\Gamma \vdash_{\equiv_{1}}^{c f+2} A, E, \Delta^{\prime}} E \equiv_{1} \neg A
$$

exists-right if the proof is $\exists_{R} \frac{\frac{\cdots}{\Gamma, \Theta \stackrel{c f+2}{\equiv_{1}} Q[t / x], \Delta}}{\Gamma, \Theta \stackrel{c f+2}{\equiv_{1}} \psi, \Delta} \psi \equiv_{1} \exists x . Q$ then by induction hypothesis we obtain a proof of $\Gamma \stackrel{c f++_{2}}{\equiv} A, Q[t / x], \Delta$ and through $\exists_{R}$ we get a proof of $\Gamma \stackrel{c f+_{1}+_{2}}{\equiv} A, \psi, \Delta$.

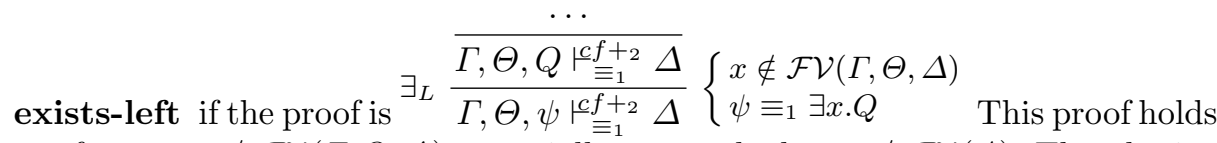
for any $x \notin \mathcal{F} \mathcal{V}(\Gamma, \Theta, \Delta)$, especially one such that $x \notin \mathcal{F} \mathcal{V}(A)$. Then by induction hypothesis we obtain a proof of $\Gamma, Q \stackrel{c f}{\stackrel{f}{\equiv}+_{2}} A, \Delta$ and through $\exists_{L}$ we get a proof of $\Gamma, \psi \stackrel{c f++_{2}}{={ }_{1}} A, \Delta$.

superdeduction-right if the proof is

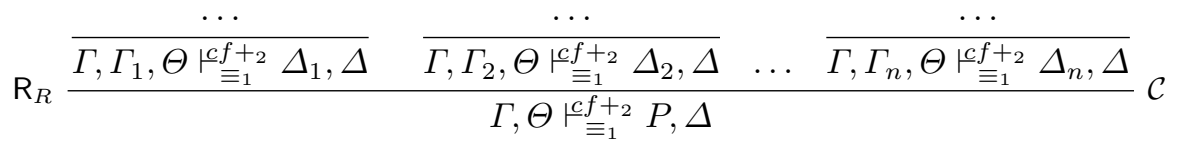

with $\mathrm{R}: P \rightarrow \varphi \in \mathcal{T} h_{2}$. Let us remark that since the variables concerned with the side condition $\mathcal{C}$ do not appear in $\mathcal{F} \mathcal{V}(\Gamma, \Theta, \Delta)$ (because $\mathcal{C}$ holds), they can even be chosen such that they do not appear in $\mathcal{F} \mathcal{V}(A)$. Then by induction hypothesis we obtain proofs of the $\left(\Gamma, \Gamma_{i} \stackrel{c}{\stackrel{c f+}{\equiv_{1}}} A, \Delta_{i}, \Delta\right)_{i}$ for $1 \leqslant i \leqslant n$ and therefore through $\mathrm{R}_{R}$ we obtain a proof of $\Gamma \stackrel{c f+{ }_{2}}{\equiv{ }_{1}} A, P, \Delta$ (in particular the side condition of this instance of $R_{R}$ is verified). 
other cases are handled the same way.

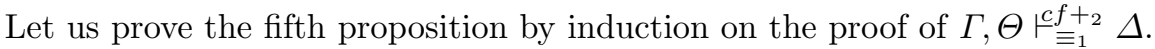
If the last step of the proof of $\Gamma, \Theta \stackrel{c f \mid c+2}{\equiv_{1}} \Delta$ decomposes $A_{i} \equiv_{1} \exists x . Q$ (we suppose without loss of generality that $i=1$ ), then since $\mathcal{T} h_{1}$ is confluent and only rewrites atomic propositions and first-order terms, the decomposition of $A_{1}$ is $\exists_{L}$, and we have a proof of $\Gamma, Q^{\prime}[c / x], A_{2} \ldots A_{n} \stackrel{c f+_{\equiv_{1}}}{f_{1}} \Delta$ for $Q \equiv_{1} Q^{\prime}$ and for some fresh variable $c$. Therefore by induction hypothesis, we obtain a proof of $\Gamma, Q^{\prime}[c / x], Q[d / x] \vdash_{\equiv=1}^{c f+2} \Delta . c$ and $d$ being two distinct fresh variables, this latter proof also holds if we replace them by another fresh variable $e$. Therefore we obtain a proof of $\Gamma, Q^{\prime}[e / x], Q[e / x] \stackrel{c f+_{2}}{\models_{1}} \Delta$. Using a contraction (since $Q^{\prime}[e /$ $\left.x] \equiv{ }_{1} Q[e / x]\right)$, we get a proof of $\Gamma, Q[e / x] \stackrel{c f+_{\equiv_{2}}}{\perp_{1}} \Delta$. Otherwise let us consider the inference rule applied at the root of the proof of $\Gamma, \exists x . Q \stackrel{c f++_{2}}{\equiv_{1}} \Delta$.

contraction the contraction may be applied on a formula of $\Theta, \Gamma$ or $\Delta$, but in all cases it is sufficient to apply the induction hypothesis on the premiss of the contraction.

axiom if the proof is $\operatorname{Ax} \overline{\Gamma, \Theta \stackrel{c f+{ }_{\equiv}}{\equiv_{1}} \Delta}$ then there exists $B \in \Gamma \cup \Theta$ and $C \in \Delta$ such that $B \equiv{ }_{1} C$. If $B \in \Gamma$ then we have a trivial proof of $\Gamma, Q[c / x] \vdash_{\equiv_{1}}^{c f+_{2}} \Delta$.

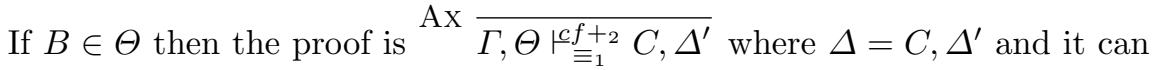
be transformed into

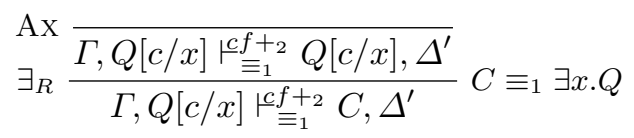

exists-right if the proof is $\exists_{R} \frac{\overline{\Gamma, \Theta \stackrel{c f+2}{\equiv_{1}} P[t / y], \Delta}}{\Gamma, \Theta \vdash_{\equiv_{1}^{c f+2}}^{++_{2}} \psi, \Delta} \psi \equiv_{1} \exists y . P$ then by induction hypothesis we obtain a proof of $\Gamma, Q[c / x] \stackrel{c f++_{2}}{\equiv_{1}} P[t / y], \Delta$ and through $\exists_{R}$ we get a proof of $\Gamma, Q[c / x] \stackrel{c f+_{2}}{\equiv} \psi, \Delta$.

exists-left if the proof is $\exists_{L} \frac{\frac{\cdots}{\Gamma, \Theta, P \stackrel{c f+2}{\equiv_{1}} \Delta}}{\Gamma, \Theta, \psi \stackrel{c f+2}{\equiv_{1}} \Delta}\left\{\begin{array}{l}y \notin \mathcal{F} \mathcal{V}(\Gamma, \Theta, \Delta) \\ \psi \equiv \equiv_{1} \exists y . P\end{array}\right.$ This proof holds for any $y \notin \mathcal{F} \mathcal{V}(\Gamma, \Theta, \Delta)$, especially one such that $y \notin \mathcal{F} \mathcal{V}(Q)$. Then by induction hypothesis we obtain a proof of $\Gamma, Q[c / x], P \stackrel{c f+_{2}}{\equiv_{1}} \Delta$ and through $\exists_{L}$ we get a proof of $\Gamma, Q[c / x], \psi \stackrel{\vdash f \equiv_{1}+_{2}}{=} \Delta$ (c being a fresh variable, we may suppose it is not $y$ and therefore $y \notin \mathcal{F} \mathcal{V}(\Gamma, Q[c / x], \Delta))$.

superdeduction-right if the proof is

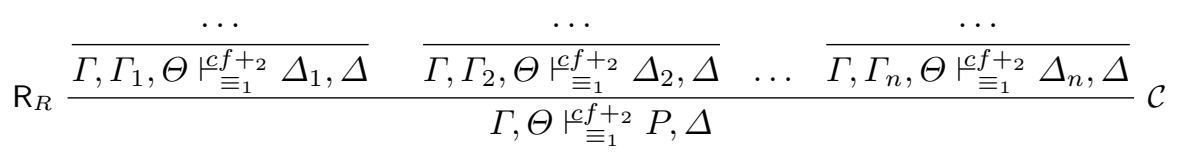


with R : $P \rightarrow \varphi \in \mathcal{T} h_{2}$. Let us remark that since the variables concerned with the side condition $\mathcal{C}$ do not appear in $\mathcal{F} \mathcal{V}(\Gamma, \Theta, \Delta)$ (because $\mathcal{C}$ holds), they can even be chosen such that they do not appear in $\mathcal{F} \mathcal{V}(Q)$. Then by induction hypothesis we obtain proofs of the $\left(\Gamma, \Gamma_{i}, Q[c / x] \stackrel{c f+2}{{ }_{\equiv}+2} \Delta_{i}, \Delta\right)_{i}$ for $1 \leqslant i \leqslant n$ and for some fresh variable $c$ and therefore through $\mathrm{R}_{R}$ we obtain a proof of $\Gamma, Q[c / x] \stackrel{c f+2}{\equiv{ }_{1}} P, \Delta$ (in particular the side condition of this instance of $\mathrm{R}_{R}$ is verified).

other cases are handled the same way.

The other propositions are proved the same way.

Then we prove the following lemma.

Lemma 10 - For some right-handed $\phi$, if there is a proof of $\Gamma \stackrel{c f++_{1}+_{1}}{\phi, \Delta \text { and }}$ $\left(\Gamma, \Gamma_{i} \stackrel{c f+2}{\equiv_{1}{ }_{1}} \Delta_{i}, \Delta\right)_{i}$ is a list of sequents representing a step of the decomposition using rule of Calc of $\phi$ in $\Gamma \stackrel{c f+_{2}}{\equiv_{1}} \phi, \Delta$, then for all $i$, there is a proof of $\Gamma, \Gamma_{i} \stackrel{c f++_{2}}{\equiv_{1}} \Delta_{i}, \Delta$.

- For some left-handed $\phi$, if there is a proof of $\Gamma, \phi \stackrel{c f+_{2}}{\equiv_{1}} \Delta$ and $\left(\Gamma, \Gamma_{i} \stackrel{c f+2}{\equiv_{1}}\right.$ $\left.\Delta_{i}, \Delta\right)_{i}$ is a list of sequents representing a step of the decomposition using rule of Calc of $\phi$ in $\Gamma, \phi \stackrel{c f++_{2}}{\equiv_{1}} \Delta$, then for all $i$, there is a proof of $\Gamma, \Gamma_{i} \stackrel{c f++_{2}}{\equiv_{1}}$ $\Delta_{i}, \Delta$.

Proof. Let us prove the first proposition. By induction on the number of steps

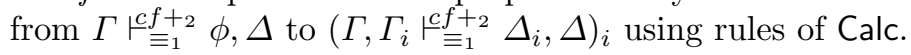

- If the list of sequents contains only $\Gamma \stackrel{c f}{\stackrel{f}{\equiv}+_{1}} \phi, \Delta$, by hypothesis there is a proof of $\Gamma \stackrel{c f}{\stackrel{f}{\equiv}+_{1}} \phi, \Delta$.

- If the step $\left(\Gamma, \Gamma_{i} \stackrel{c f}{\stackrel{c f++_{2}}{\equiv_{1}}} \Delta_{i}, \Delta\right)_{i}$ is obtained from previous step $\left(\Gamma, \Gamma_{k}^{\prime} \stackrel{c f+_{2}}{\equiv_{1}}\right.$ $\left.\Delta_{k}^{\prime}, \Delta\right)_{k}$ using the rule

axiom then all the sequents $\Gamma, \Gamma_{i} \stackrel{c f}{\stackrel{c f+2}{\equiv}{ }_{1}} \Delta_{i}, \Delta$ are already in the list $\left(\Gamma, \Gamma_{k}^{\prime} \stackrel{c f+2}{\models_{1}+_{2}}\right.$ $\left.\Delta_{k}^{\prime}, \Delta\right)_{k}$. Therefore by induction hypothesis, for all $i$ there exists a proof of $\Gamma, \Gamma_{i} \stackrel{\iota f}{\stackrel{f+}{\equiv}+_{1}} \Delta_{i}, \Delta$.

implication left then all the sequents $\Gamma, \Gamma_{i} \stackrel{c f}{\stackrel{c f++_{2}}{\equiv}} \Delta_{i}, \Delta$ are already in the list $\left(\Gamma, \Gamma_{k}^{\prime} \stackrel{c f+_{2}}{\equiv+_{1}} \Delta_{k}^{\prime}, \Delta\right)_{k}$ except some sequents $\Gamma, \Gamma^{\prime}, \psi \stackrel{c f+_{2}}{\equiv_{1}} \Delta^{\prime}, \Delta$ and $\Gamma, \Gamma^{\prime} \stackrel{c f+_{2}}{\equiv_{1}} \varphi, \Delta^{\prime}, \Delta$ that comes from decomposing $\varphi \Rightarrow \psi$ in $\Gamma, \Gamma^{\prime}, \varphi \Rightarrow$ $\psi \stackrel{c f+_{1}}{\vdash_{1}} \Delta^{\prime}, \Delta$. From induction hypothesis, there exists proofs of all sequents from the previous step. Therefore there exists a proof of $\Gamma, \Gamma^{\prime}, \varphi \Rightarrow$ $\psi \stackrel{c f+_{2}}{\digamma_{1}} \Delta^{\prime}, \Delta$. By Lemma 3, we obtain proofs of $\Gamma, \Gamma^{\prime}, \psi \stackrel{c f \risingdotseq_{1}+_{2}}{=} \Delta^{\prime}, \Delta$ and $\Gamma, \Gamma^{\prime} \stackrel{c f+_{2}}{\equiv} \varphi, \Delta^{\prime}, \Delta$ and consequently we have proofs of all sequents $\left(\Gamma, \Gamma_{i} \stackrel{c}{\stackrel{c}{\bar{f}++_{2}} \Delta_{i}} \Delta_{i}, \Delta\right)$.

forall right then all the sequents $\Gamma, \Gamma_{i} \stackrel{c f}{\stackrel{c f+2}{\equiv}{ }_{1}} \Delta_{i}, \Delta$ are already in the list $\left(\Gamma, \Gamma_{k}^{\prime} \vdash_{\equiv}^{c f+_{2}} \Delta_{k}^{\prime}, \Delta\right)_{k}$ except some sequent $\Gamma, \Gamma^{\prime} \vdash_{\equiv_{1}}^{c f+_{2}} \psi[c / x], \Delta^{\prime}, \Delta$ that comes from decomposing $\forall x . \psi$ in $\Gamma, \Gamma^{\prime} \stackrel{c f{ }_{\equiv}+_{2}}{1} \forall x . \psi, \Delta^{\prime}, \Delta$ where $c$ is a fresh variable. By induction hypothesis, there exists proofs of all sequents from the previous step. Therefore there exists a proof of $\Gamma, \Gamma^{\prime} \stackrel{c f+2}{\equiv_{1}}$ 
$\forall x . \psi, \Delta^{\prime}, \Delta$. By Lemma 3, we obtain a proof of $\Gamma, \Gamma^{\prime} \stackrel{c f++_{2}}{\stackrel{\equiv}{1}_{1}} \psi[c / x], \Delta^{\prime}, \Delta$

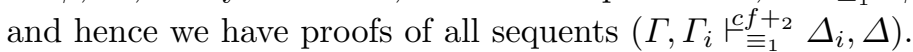
exists right This case is contradictory since $\phi$ is right-handed. forall left This case is contradictory since $\phi$ is right-handed. exists left This case is contradictory since $\phi$ is right-handed. other cases are handled the same way.

The second proposition is symmetrical.

Let us prove now Lemma 4.

Lemma 4 Let us consider some $\mathrm{R}: P \rightarrow \varphi \in \mathcal{T} h_{2}$.

- If $\varphi$ is right-handed and $\Gamma \stackrel{c f++_{2}}{\stackrel{\equiv_{1}}{1}} \varphi, \Delta$, then there exists (cut-free) proofs of each premise of the introduction of $P$ on the right. Therefore $\Gamma \stackrel{c f++_{2}}{\equiv_{1}} P, \Delta$.

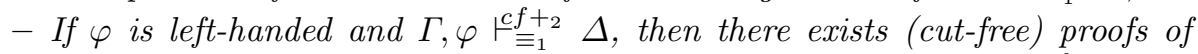

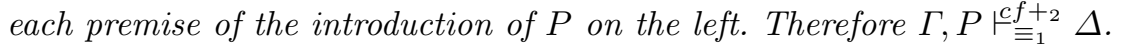

Proof. Let us prove the first proposition: Since there exists a proof of $\Gamma \stackrel{c f+2}{\equiv} \stackrel{1}{\equiv}_{1}$ $\varphi, \Delta$, by Lemma 10 and since the premises of the $\mathrm{R}_{R}$ rule represent a step (the last one) of the decomposition of $\varphi$ in $\Gamma \stackrel{\stackrel{c f++_{2}}{\equiv} \equiv_{1}}{,}, \Delta$, by Lemma 10 there exists proofs of each premise (where the variables appearing in the side condition of $\mathrm{R}_{R}$ are replaced by fresh variables: consequently this side condition is verified). Therefore through $\mathrm{R}_{R}$ we obtain a proof of $\Gamma \stackrel{c f+\stackrel{c}{\equiv}_{1}}{=} P, \Delta$.

The second proposition is proved in a symmetrical manner.

Before proving Lemma 5, we prove this auxiliary lemma:

Lemma 11 Let us consider $\mathrm{R}: P \rightarrow \varphi$.

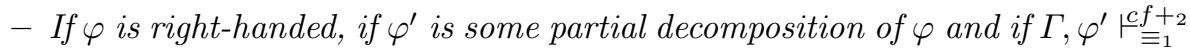
$\Delta$, then there is a proof of $\Gamma, P \stackrel{c f++_{2}}{\stackrel{\equiv}{=}_{1}} \Delta$.

- If $\varphi$ is left-handed, if $\varphi^{\prime}$ is some partial decomposition of $\varphi$ and if $\Gamma \stackrel{c f++_{2}}{\stackrel{1}{1}_{1}}$ $\varphi^{\prime}, \Delta$, then there is a proof of $\Gamma \stackrel{c f+{ }_{\equiv}+_{1}}{=} P, \Delta$.

Proof. Let us prove the first proposition. First by Lemmas 7, 8, 9, we can suppose that $\varphi^{\prime}$ is fully decomposed and non-contracted in the proof. Then we proceed by induction on this proof.

axiom on $\varphi^{\prime}$ : The proof is $\operatorname{Ax} \overline{\Gamma, \varphi^{\prime} \stackrel{c f+2}{\models_{1}} \psi, \Delta^{\prime}} \varphi^{\prime} \equiv_{1} \psi$ Since $\varphi^{\prime}$ is fully decomposed in the proof, it is then an atomic predicate $(=\phi \sigma$ for some $\sigma)$. Then the $\mathrm{R}_{L}$ rule is $\mathrm{R}_{L} \frac{\Gamma, \phi \rho \vdash \Delta}{\Gamma, P \vdash \Delta}$ (for any substitution $\rho$ ) and then the following proof holds.

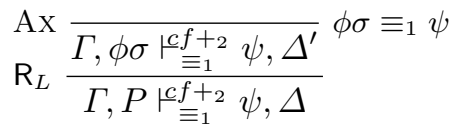


axiom on $\Gamma, \Delta$. This case is obvious.

contraction on $\Gamma, \Delta$. This case is obvious.

contraction on $\varphi^{\prime}$. Since $\varphi^{\prime}$ is non-contracted in the proof, it can only be a propositional formula $\phi \sigma$. Then by Lemma 4 , there are proofs of each premise $\Gamma, \Gamma_{i} \sigma \stackrel{c f++_{2}}{={ }_{1}} \Delta_{i} \sigma, \Delta$ of a decomposition of $\phi \sigma$ on the left in $\Gamma, \varphi^{\prime} \stackrel{c f++_{2}}{\stackrel{\equiv}{=}} \Delta$. Besides the $\mathrm{R}_{L}$ inference rule is

$$
\mathrm{R}_{L} \frac{\left(\Gamma, \Gamma_{i} \rho \vdash \Delta_{i} \rho, \Delta\right)_{i}}{\Gamma, P \underset{\equiv_{1}^{c f+2}}{\equiv} \Delta}
$$

(for any substitution $\rho$ ). Then through $\mathrm{R}_{L}$ we get a proof of $\Gamma, P \stackrel{c f f+2}{\models_{1}} \Delta$.

decomposition of a formula of $\Gamma, \Delta$. We simply apply the induction hypothesis on each premise.

decomposition of $\varphi^{\prime}=\phi \sigma$ : This case is handled the same way as a contraction on $\varphi^{\prime}=\phi \sigma$.

decomposition of $\varphi^{\prime}=\forall x_{k} \ldots x_{n} . \phi \sigma$ :

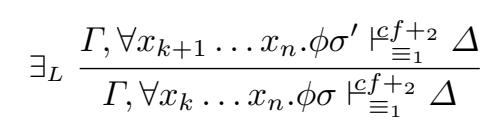

Then by induction hypothesis, there exists a proof of $\Gamma, P \stackrel{\stackrel{c f++_{2}}{\equiv}{ }_{1}}{ } \Delta$.

Since the second proposition is symmetrical, it is proved in a similar way.

Let us prove now Lemma 5 .

Lemma 5 Let us consider $\mathrm{R}: P \rightarrow \varphi \in \mathcal{T h}_{2}$.

- If $\varphi$ is right-handed and $\Gamma, \varphi \stackrel{c f++_{2}}{\equiv_{1}} \Delta$, then $\Gamma, P \stackrel{c f++_{2}}{\equiv_{1}} \Delta$.

- If $\varphi$ is left-handed and $\Gamma \stackrel{c f++_{2}}{\stackrel{\equiv_{1}}{1}} \varphi, \Delta$, then $\Gamma \stackrel{c f++_{2}}{\stackrel{\equiv_{1}}{I}} P, \Delta$.

Proof. If $\varphi$ is right-handed (resp. left-handed), then $\varphi$ is a partial decomposition of itself and consequently by Lemma 11, if there is a proof of $\Gamma, \varphi \stackrel{c f++_{2}}{\equiv_{1}} \Delta$ (resp.

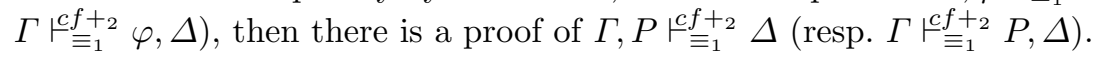

